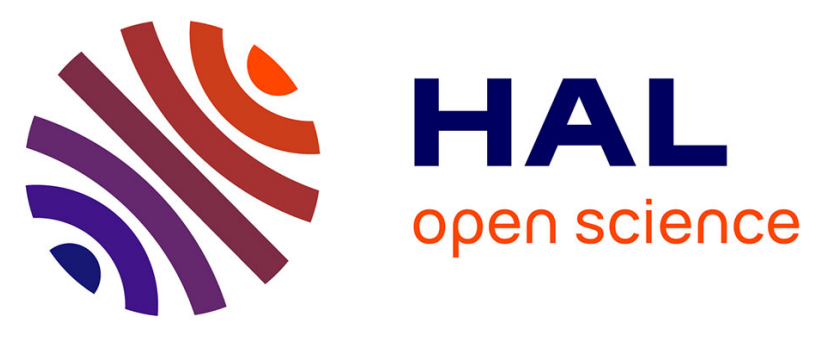

\title{
Conducting chiral nickel( ii ) bis(dithiolene) complexes: structural and electron transport modulation with the charge and the number of stereogenic centres
}

Alexandre Abhervé, Nabil Mroweh, Thomas Cauchy, Flavia Pop, Hengbo Cui, Reizo Kato, Nicolas Vanthuyne, Pere Alemany, Enric Canadell, Narcis Avarvari

\section{To cite this version:}

Alexandre Abhervé, Nabil Mroweh, Thomas Cauchy, Flavia Pop, Hengbo Cui, et al.. Conducting chiral nickel( ii ) bis(dithiolene) complexes: structural and electron transport modulation with the charge and the number of stereogenic centres. Journal of Materials Chemistry C, 2021, 9 (12), pp.4119-4140. 10.1039/D1TC00439E . hal-03452227

\section{HAL Id: hal-03452227 \\ https://univ-angers.hal.science/hal-03452227}

Submitted on 26 Nov 2021

HAL is a multi-disciplinary open access archive for the deposit and dissemination of scientific research documents, whether they are published or not. The documents may come from teaching and research institutions in France or abroad, or from public or private research centers.
L'archive ouverte pluridisciplinaire HAL, est destinée au dépôt et à la diffusion de documents scientifiques de niveau recherche, publiés ou non, émanant des établissements d'enseignement et de recherche français ou étrangers, des laboratoires publics ou privés. 


\title{
Conducting chiral nickel(II) bis(dithiolene) complexes: structural and electron transport modulation with the charge and the number of stereogenic centres
}

\author{
Alexandre Abhervé, ${ }^{\mathrm{a}}$ Nabil Mroweh, ${ }^{\mathrm{a}}$ Thomas Cauchy, ${ }^{\mathrm{a}}$ Flavia Pop, ${ }^{\mathrm{a}}$ HengBo Cui, ${ }^{\mathrm{b}}$ Reizo Kato, ${ }^{\mathrm{b}}$ \\ Nicolas Vanthuyne, ${ }^{c}$ Pere Alemany, ${ }^{d}$ Enric Canadell ${ }^{*, e}$ and Narcis Avarvari*,a
}

\author{
${ }^{a}$ MOLTECH-Anjou, UMR 6200, CNRS, UNIV Angers, 2 bd Lavoisier, 49045 ANGERS Cedex, France. E- \\ mail: narcis.avarvari@univ-angers.fr \\ ${ }^{b}$ Condensed Molecular Materials Laboratory, RIKEN, 2-1 Hirosawa, Wako, Saitama 351-0198, Japan \\ ${ }^{c}$ Aix Marseille Université, CNRS, Centrale Marseille, iSm2, Marseille, France \\ ${ }^{d}$ Departament de Ciència de Materials i Química Física i Institut de Química Teórica i Computacional \\ (IQTCUB), Universitat de Barcelona, Martí i Franquès 1, 08028 Barcelona, Spain \\ e Institut de Ciència de Materials de Barcelona, ICMAB-CSIC, Campus de la UAB, E-08193, Bellaterra, \\ Spain. E-mail: canadell@icmab.es
}

\begin{abstract}
Nickel(II) bis(dithiolene) complexes can provide crystalline conducting materials either in their monoanionic or neutral forms. Here we show that the use of chiral dithiolene ligands with one or two stereogenic centres, together with variation of the counter-ion in the anionic complexes, represents a powerful strategy to modulate the conducting properties of such molecular materials. The chiral ligands 5-methyl-5,6-dihydro-1,4-dithiin-2,3-dithiolate (me-dddt) and 5,6-dimethyl-5,6-dihydro-1,4dithiin-2,3-dithiolate ( $\mathbf{d m}$ - $d d d t$ ) have been generated from the thione precursors $\mathbf{1}$ and $\mathbf{2}$ which have been structurally and chiroptically characterized. Anionic $\mathrm{Ni}(\mathrm{II})$ complexes of these two ligands with tetrabutyl-ammonium (TBA) and tetramethyl-ammonium (TMA) have been prepared and structurally characterized, suggesting that it is the nature of the counter-ion which mostly influences the solid state organization of the complexes. Both TBA and TMA radical anion salts are Mott insulators with antiferromagnetic ground state, as suggested by spin polarized DFT band structure calculations. However, the TMA salts are one order of magnitude more conducting than the TBA counterparts. The neutral materials [ $\mathbf{N i ( m e - d d d t})_{2}$ ] and [ $\left.\mathbf{N i ( d m - d d d t}\right)_{2}$ ] are direct band gap semiconductors, as determined by DFT and extended Hückel band structure calculations, with their conductivity drastically increased up to $0.05-3.3 \mathrm{~S} \mathrm{~cm}^{-1}$ under the highest applied pressures of $10-11 \mathrm{GPa}$. At equivalent applied pressures the dm-dddt materials are more conducting than the me-dddt ones, in agreement with the lower calculated activation energy and higher bands dispersion for the former. This trend follows the structural change when going from one to two methyl substituents, since the packing and intermolecular interactions are completely different between [ $\left.\mathbf{N i}(\mathbf{d m}-\mathbf{d d d t})_{2}\right]_{\text {and }}\left[\mathrm{Ni}(\mathrm{me}-\mathbf{d d d t})_{2}\right]_{\text {, the }}$ packing of the latter being related to the one of the achiral parent [Ni(dddt) $)_{2}$. Subtle differences of conductivity are also observed within both series of neutral complexes between the enantiopure and racemic forms. This represents the first series of chiral nickel bis(dithiolene) complexes which shows modulation of the conducting properties with the number of stereogenic centres, the conductivity, measured on single crystals, strongly increasing upon applying hydrostatic pressure.
\end{abstract}




\section{Introduction}

Metal-bis(dithiolene) complexes ${ }^{1}$ represent an important family of electroactive precursors for conducting materials ${ }^{2,3}$ thanks to the non-innocent nature of the unsaturated dithiolate ligand, ${ }^{4,5}$ triggering reversible redox processes and thus allowing the access to stable anionic, neutral and even cationic species. ${ }^{6,7}$ Open-shell compounds with divalent metals such as $\mathrm{Ni}(I I)$, providing square-planar complexes favouring efficient stacking and orbital overlap in the solid state, ${ }^{2,3}$ are readily accessible as monoanionic complexes upon aerial one-electron oxidation of the initially generated dianionic complexes. ${ }^{1,8}$ Further one-electron chemical or electrochemical oxidation can provide closed-shell neutral species [Ni(dithiolene $\left.)_{2}\right]^{7}$ as single-component conductors which, depending on the ligand backbone and substitution pattern, are endowed with various conducting properties. Indeed, $\mathrm{Ni}(\mathrm{II})$ complexes containing tetrathiafulvalene (TTF) dithiolene ligands ${ }^{9}$ show under ambient pressure either metallic conductivity, such as [ $\left.\mathrm{Ni}(\mathrm{tmdt})_{2}\right]\left(\mathrm{tmdt}=\right.$ trimethylene-TTF-dithiolate), ${ }^{10,11}$ or semiconducting behaviour as observed, for example, for $\left[\mathrm{Ni}-\left(\mathrm{MeS}_{2} \mathrm{dt}\right)_{2}\right]\left(\mathrm{MeS}_{2} \mathrm{dt}=\right.$ dimethylthio-TTF-dithiolate), ${ }^{12}$ $\left[\mathrm{Ni}(\mathrm{ptdt})_{2}\right](\mathrm{ptdt}=\text { propylenedithio-TTF-dithiolate })^{13}$ or $\left[\mathrm{Ni}(\mathrm{hfdt})_{2}\right](\mathrm{hfdt}=$ bis(trifluoromethyl)-TTFdithiolate).${ }^{14}$ However, upon applied hydrostatic pressure of $\approx 19 \mathrm{GPa}$, [Ni(ptdt) 2 ] was shown to transit to a metallic regime, ${ }_{15}^{15}$ while $\left[\mathrm{Ni}(\mathrm{hfdt})_{2}\right.$ ] even showed superconducting transition for pressures of 7.5 8.7 $\mathrm{GPa} .{ }^{16}$ The critical role of the pressure has been evidenced as well in the case of neutral [Ni(dithiolene) $)_{2}$ ] complexes without TTF units on the ligand backbone, in particular in the case of [Ni(dmit) $)_{2}$ (dmit = 1,3-dithiole-2-thione-4,5-dithiolate) which became metallic above $15.9 \mathrm{GPa},{ }^{17}$ or triclinic [Ni(Et-thiazdt) ${ }_{2}$ (Et-thiazdt $=\mathrm{N}$-ethyl-1,3-thiazoline-2-thione-4,5-dithiolate) which reached a quasi-metallic state at $6.9 \mathrm{GPa}^{18}$ However, other neutral [Ni(dithiolene) ${ }_{2}$ ] complexes, such as $\left[\mathrm{Ni}(\mathrm{dddt})_{2}\right]$ (dddt $=$ 5,6-dihydro-1,4-dithiin-2,3-dithiolate) ${ }^{19}$ (Scheme 1 ) or monoclinic [Ni(Etthiazdt $\left.)_{2}\right],{ }^{20}$ remained semiconducting under pressure although their conductivity dramatically increased. The case of the former is particularly intriguing since, although it is isostructural with its $\left[\mathrm{Pd}(\mathrm{dddt})_{2}\right]$ analogue which shows characteristics of Dirac-cone material at high pressure, such as temperature-independent resistivity at $12.6 \mathrm{GPa}$ and emergence of a nodal line semimetal in the band structure under pressure, ${ }^{21,22,23}$ it remains semiconductor up to the highest applied pressure. Moreover, the activation energy $\mathrm{E}_{\mathrm{a}}$ and temperature-dependent resistivity values of [Ni(dddt $\left.)_{2}\right]$ show pressure independence in the range $7.3 \mathrm{GPa}$ to $21.6 \mathrm{GPa}$ and between 10.9 and $21.6 \mathrm{GPa}$, respectively, the room temperature conductivity at the highest pressures being 1.1-1.2 $\mathrm{S} \mathrm{cm}^{-1}$ with an activation energy of $43-46 \mathrm{meV} .{ }^{19}$ This shows that subtle chemical changes in the structure of single component conductors, here the replacement of $\mathrm{Pd}(\mathrm{II})$ with $\mathrm{Ni}(\mathrm{II})$, can lead to completely different conducting properties. A straightforward strategy to modulate the crystal packing and intermolecular interactions in the field of molecular conductors is the introduction of chirality, ${ }^{24}$ which has been particularly investigated so far in the case of chiral TTF based conductors. ${ }^{25}$ Besides its direct influence on the conducting properties, referred to as electrical magnetochiral anisotropy (eMChA) effect, ${ }^{26,27,28}$ chirality has been shown to strongly influence the conducting properties through the modulation of the structural disorder, ${ }^{29,30,31}$ or to provide completely different packing, and thus conducting properties, upon using enantiopure or racemic TTF precursors. ${ }^{32,33}$ Conducting chiral metal-dithiolene complexes have been, comparatively, much less investigated. ${ }^{34,35,36}$ Moreover, no systematic study on the influence of the number of stereogenic centres on the conducting properties of anionic and neutral metal bis(dithiolene) complexes has been performed to date. Since the presence of dimethylethylenedithio (dm-edt) or methyl-ethylenedithio (me-edt) units in the structure of chiral TTF precursors such as DM-EDT-TTF, ${ }^{27,32,37}$ DM-BEDT-TTF, ${ }^{38}$ or Me-EDT-TTF ${ }^{39}$ proved to be beneficial for the 
observation of a rich variety of structural variations and conducting properties, we decided to investigate the introduction of one or two methyl substituents on the dddt ligand in the corresponding chiral anionic and neutral $\mathrm{Ni}$ (dithiolene) ${ }_{2}$ complexes (Scheme 1 ), following our previous report dealing with the synthesis and properties of enantiopure $\left(\mathrm{NBu}_{4}\right)\left[\mathrm{M}(\mathrm{dm} \text {-dddt })_{2}\right]$ and $\left[\mathrm{M}(\mathrm{dm} \text {-dddt })_{2}\right](\mathrm{M}=\mathrm{Ni}, \mathrm{Au})$ complexes. ${ }^{40}$ While only the conducting properties of the $\left(\mathrm{NBu}_{4}\right)\left[\mathrm{Ni}(\mathrm{dm} \text {-dddt })_{2}\right]$ salts have been previously investigated at ambient pressure, we present herein an in-depth study on the modulation of the crystal structures and transport properties under high pressure with the charge and number of stereogenic centres in the anionic and neutral nickel-bis(dithiolene) complexes containing the dm-dddt and me-dddt ligands, together with extended Hückel and DFT band structure calculations. The present study is the first one reporting the use of the ligand me-dddt, containing only one stereogenic centre. Its lack of $C_{2}$ symmetry, compared to the dimethylated analogue dm-dddt, allows, in principle, the existence of cis and trans isomers for corresponding bis(dithiolene) complexes (Scheme 1). Moreover, the influence of the counterion, i.e. $\left(\mathrm{NBu}_{4}\right)^{+}$versus the smaller $\left(\mathrm{NMe}_{4}\right)^{+}$, on the crystal structures and hence the conducting properties of the anionic complexes, has been addressed as well.

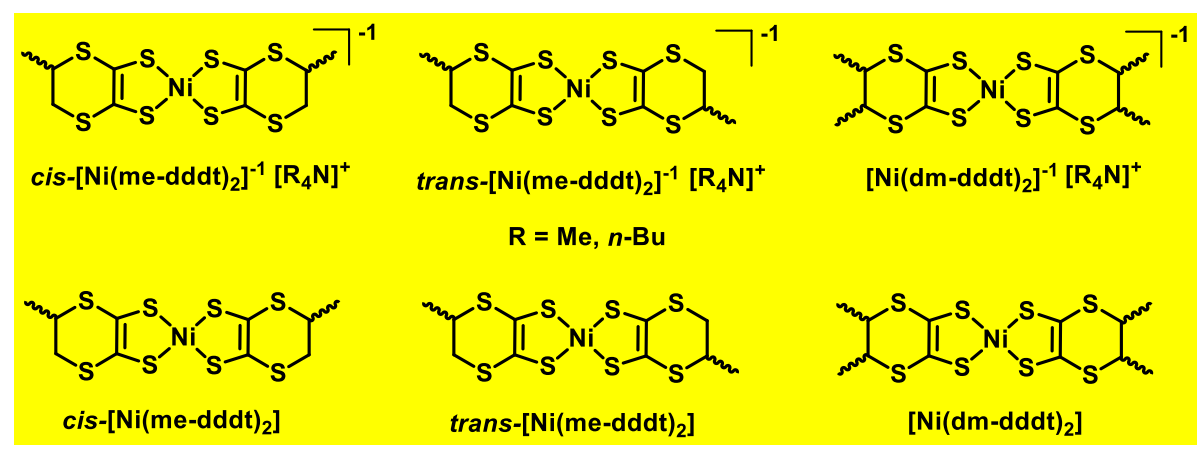

Scheme 1 Chiral methylated anionic and neutral $\mathrm{Ni}$ (II) bis-dithiolene complexes derived from the dddt ligand.

\section{Results and discussion}

\subsection{Synthesis, chiroptical properties and crystal structures of the dithiolethione precursors}

The thione precursors me-dddt-thione (1) and dm-dddt-thione (2) (Scheme 2) were prepared as racemic mixtures. Accordingly, precursor $\mathbf{1}$ was synthesized following the procedure recently described by us, ${ }^{39}$ while for precursor $\mathbf{2}$ we have adapted the protocol reported by Papavassiliou et al., ${ }^{41}$ involving a hetero Diels-Alder [4+2] cycloaddition between 1,3-dithiole-2,4,5-trithione with trans2 -butene, by performing the reaction under pressure and thus reaching up to $70 \%$ yield instead of $29 \%$ reported in the original procedure (see the ESI). Note that dm-dddt-thione $\mathbf{2}$ can be also obtained directly in enantiopure forms, yet involving a multistep reaction sequence. ${ }^{32,42,43}$ Both compounds (rac)-1 and (rac)-2 have been separated as pure enantiomers by semi-preparative chiral HPLC on Chiralpak IF $(250 \times 10 \mathrm{~mm})$ and on Chiralpak ID $(250 \times 10 \mathrm{~mm})$, respectively (Fig. S1-S3 for 1 and Fig. S4-S6 for 2, ESI). Since optical rotations and circular dichroism (CD) spectra (ESI) are important chiroptical characteristics, they were determined for both pairs of enantiomers. Accordingly, the absolute configurations have been attributed by comparison of the CD spectra with those of Me-EDTTTF $^{39}$ and DM-EDT-TTF, ${ }^{44}$ and confirmed by single crystal X-ray diffraction analysis on $(S, S)-2$ and $(R, R)$ 2 (vide infra). As for the corresponding Me-EDT-TTF ${ }^{39}$ and DM-EDT-TTF ${ }^{44}$ derivatives, $(S)-\mathbf{1}$ and $(S, S)-\mathbf{2}$ enantiomers are levorotatory, with specific optical rotation values of -48 and $-371 \mathrm{deg} \cdot \mathrm{mL} \cdot \mathrm{g}^{-1} \cdot \mathrm{dm}^{-1}$ 
$\left([\alpha]_{D}{ }^{25}\right.$ in $\left.\mathrm{CH}_{2} \mathrm{Cl}_{2}\right)$, respectively, while opposite values have been measured for $(R)-\mathbf{1}$ and $(R)$-2. Mirrorimage CD spectra have been obtained for both $(S)-\mathbf{1} /(R)-\mathbf{1}$ and $(S, S)-\mathbf{2} /(R, R)-\mathbf{2}$ (Fig. S7-S8, ESI). While the common feature of the CD spectra of $(S)-\mathbf{1}$ and $(S, S)-\mathbf{2}$ consists of an intense negative band peaking at $263 \mathrm{~nm}$ and $270 \mathrm{~nm}$ (positive bands for the $(R)$ and $(R, R)$ enantiomers), respectively, the observed remaining bands show, somehow surprisingly, opposite signs. Indeed, besides the negative band at $263 \mathrm{~nm},(S)-1$ presents an intense negative band at $206 \mathrm{~nm}$, a week positive band at $311 \mathrm{~nm}$ and an intense negative/positive bisignate band at $404 / 438 \mathrm{~nm}$, whereas $(S, S)-2$ shows a very week negative band at $193 \mathrm{~nm}$, followed by a structured intense positive band between 215 and $250 \mathrm{~nm}$, the negative band at $270 \mathrm{~nm}$ previously mentioned, and then an intense bisignate positive/negative band at $401 / 438 \mathrm{~nm}$. Particularly interesting is the change of sign of the bisignate band for $\mathbf{1}$ and $\mathbf{2}$, very likely caused by the preponderance in solution in the case of me-dddt 1 of the equatorial (eq) conformer with respect to the orientation of the methyl substituent, while in dm-dddt $\mathbf{2}$ the major conformer should be axial (ax,ax), when considering the calculations performed on DM-EDT-TTF. ${ }^{44}$

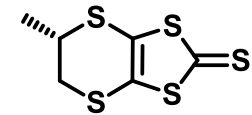

$(S)-1$

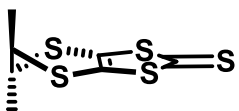

$(S, S)-2(a x, a x)$

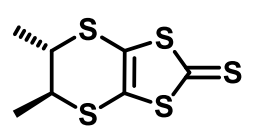

$(S, S)-2$

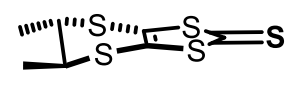

$(S, S)-2$ (eq,eq)

Scheme $2(S)$ and $(S, S)$ enantiomers of thione precursors $\mathbf{1}$ and $\mathbf{2}$ with a highlight on the axial-equatorial conformational equilibrium for precursor 2.

In order to assess this assumption, DFT and TD-DFT calculations have been carried out for the compounds $\mathbf{1}$ and $\mathbf{2}$ on both enantiomers and for each axial and equatorial conformations (Scheme 2 and Fig. S9-S48, ESI). The gas phase calculated energies confirm indeed a weakly favoured equilibrium for the equatorial form of the mono-methyl precursor $\mathbf{1}$, while for thione $\mathbf{2}$ the (ax,ax) conformation is more stable by $2.2 \mathrm{kcal} / \mathrm{mol}$ and thus clearly predominant over the (eq,eq) conformation (Table 1). This result represents a first important consequence of the number of stereogenic centres on the conformational stability of substituted dddt based ligands. These energy differences allow for the rationale of the experimental CD spectra discussed above. In Fig. 1 are reported the experimental spectra for $(R)-\mathbf{1}$ and $(R, R)-\mathbf{2}$ as well as the theoretical curves based on TD-DFT calculations. The bisignate bands sign depends on the conformation. Despite a slight blue shift observed between the experimental and theoretical curves, it appears that the experimental bands signs correspond to the most stable conformations. Furthermore, the weak intensity measured for compound $\mathbf{1}$ is in agreement with the eq/ax 2:1 ratio suggested by the calculations, leading to the strong attenuation of the bisignate band, while the signal of compound $\mathbf{2}$ is associated to an almost pure (ax,ax) conformation. It was also shown for DM-EDT-TTF that the interconversion energy barrier between the (ax,ax) and (eq,eq) conformations amounts to only $\approx 3 \mathrm{kcal} \mathrm{mol}^{-1}, 44$ suggesting a rapid equilibrium in solution at room temperature, as it very likely occurs as well for $\mathbf{1}$ and $\mathbf{2}$. 
Table 1 Gibbs Free Energies differences in $\mathrm{kcal} / \mathrm{mol}$ between the axial and equatorial conformations of $(R)-\mathbf{1}$ and $(R, R)-\mathbf{2}$ together with their expected ratios in the gas phase and room temperature

\begin{tabular}{lcc} 
Compound & $\Delta \mathrm{G}(\mathrm{ax}-\mathrm{eq})$ & $\begin{array}{c}\mathrm{RT} \text { ax }: \text { eq } \\
\text { gas phase ratio }\end{array}$ \\
\hline$(R)-\mathbf{1}$ & 0.4 & $1: 2$ \\
$(R, R)-\mathbf{2}$ & -2.2 & $40: 1$ \\
\hline
\end{tabular}
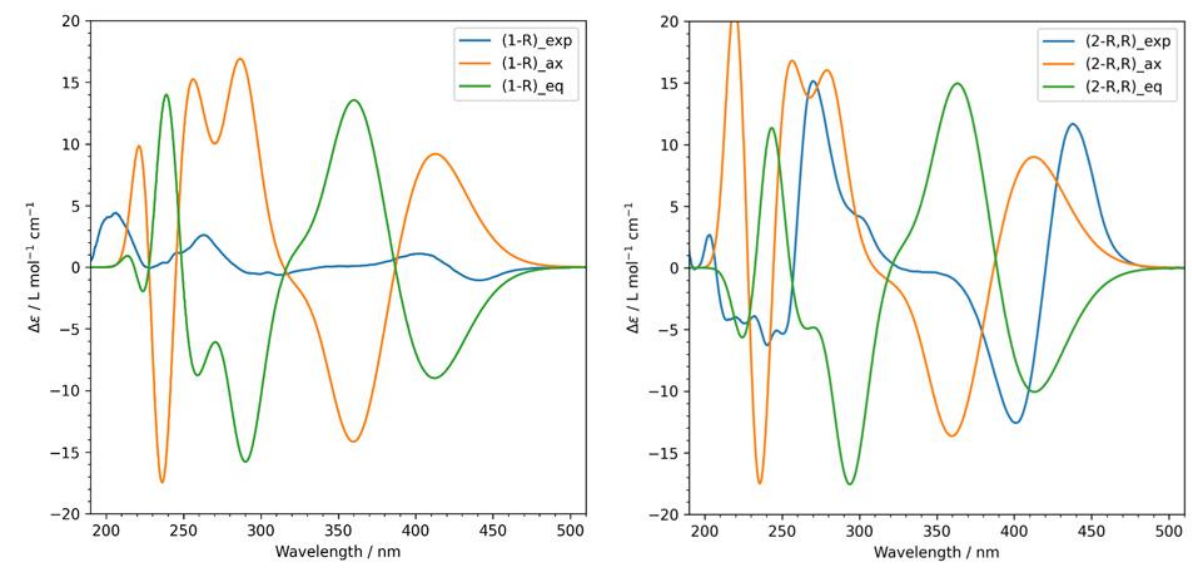

Fig. 1 CD spectra of $(R)-\mathbf{1}$ (left) and $(R, R)-\mathbf{2}$ (right). The experimental data correspond to the blue lines. The orange and green curves are respectively based on the theoretical data for the axial and equatorial conformations.

A final proof for the correct assignment of the absolute configuration was provided by the single crystal X-ray diffraction analysis (Table S1, ESI). Slow evaporation of dichloromethane (DCM) solutions of both enantiomers $(S, S)-\mathbf{2}$ and $(R, R)-\mathbf{2}$ afforded suitable single crystals. Additionally, single crystals of the racemic mixture have been also obtained by the same procedure. The pure enantiomers are isostructural and crystallize in the non-centrosymmetric monoclinic space group $P 2_{1}$, with two independent molecules in the asymmetric unit, while (rac)-2 crystallizes in the centrosymmetric space group $P 2_{1} / n$, with one independent molecule in the asymmetric unit (Fig. 2). Interestingly, in the enantiopure crystals the methyl groups adopt the (ax,ax) conformation, whereas the (eq,eq) one is observed in the racemate, which also shows disorder of the ethylene bridge (Fig. 2). Since the two conformers are in equilibrium in solution, with, very likely, the (ax,ax) conformer being the major one (vide supra), the occurrence of one or the other in the solid state depends on the packing and the crystallization conditions, as observed, for example, in the case of TM-BEDT-TTF. ${ }^{31,45}$ The crystal packing is also different between the enantiopure and racemic forms of $\mathbf{2}$, with a criss-cross stacking pattern along $a$ for the former and formation of staggered centrosymmetric dyads for the latter (Fig. S49, ESI). Note that Wallis and Dunitz have previously reported a pseudo-centrosymmetric structure of $(S, S)-2$, isostructural of $(r a c)-2$ we describe herein, in which the two methyl substituents arrange in equatorial positions. ${ }^{46}$

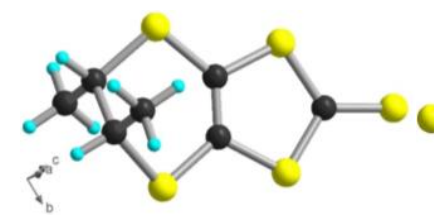

a)

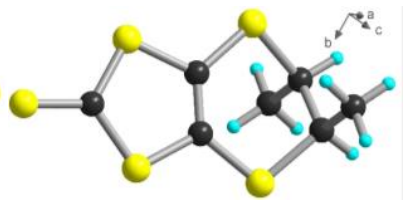

b) 


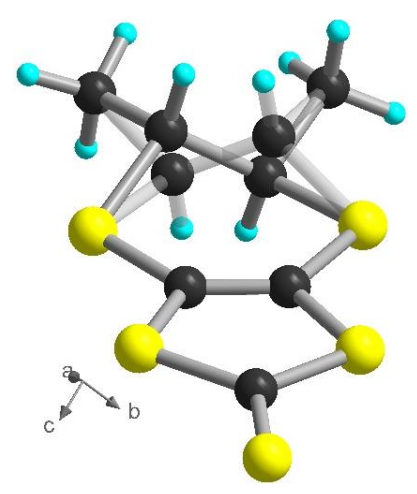

c)

Fig. 2 Crystal structure of the precursors $(S, S)-2(a),(R, R)-2(b)$ and $(r a c)-2(c)$.

Finally, the racemic and enantiomerically pure dithiolones $\mathbf{3}$ and $\mathbf{4}$, which served as precursors for the corresponding $\mathrm{Ni}$ bis(dithiolene) complexes, were prepared from the thione precursors $\mathbf{1}$ and $\mathbf{2}$, respectively, following the classical procedure of oxymercuration (Scheme 3 ). ${ }^{32}$

\subsection{Synthesis of complexes}

As already mentioned, in this work we aimed to study the influence of the chiral dithiolene ligands 5methyl-dddt (me-dddt) and 5,6-dimethyl-dddt (dm-dddt) on the conducting properties of the corresponding anionic and neutral nickel complexes. Note that the former ligand has never been used before. Moreover, in order to investigate the influence of the counterion in the anionic salts, we decided to replace the bulky tetrabutylammonium (TBA) cation by the smaller tetramethylammonium (TMA), following our previous report on $\left(\mathrm{NBu}_{4}\right)\left[\mathrm{Ni}(\mathrm{dm}-\mathrm{ddt})_{2}\right] .{ }^{40}$ To date, numerous TMA salts of $\mathrm{Ni}$ bis(dithiolene) complexes have been reported, although only two of them were based on dddt derivatives. Accordingly, the compound (TMA)[Ni(dmeo-dddt) 2 (dmeo-dddt = 5,6-dimethoxy-dddt), which crystal structure has not been described, was used as a precursor for the preparation of the neutral derivative. ${ }^{47}$ For the compound $(\mathrm{TMA})\left[\mathrm{Ni}(\mathrm{dddt})_{2}\right]$, reported back in $1989,{ }^{48}$ structural and magnetic characterization confirmed the enhanced intermolecular interactions between anionic complexes in the TMA salt over the TBA analogue.

The (TBA) and (TMA) salts of the anionic nickel-bis(dithiolene) complexes were prepared following the classical reported procedure involving deprotection of the thione precursors with sodium methanolate, reaction with $\mathrm{NiCl}_{2}$ and cation metathesis (Scheme 3). ${ }^{40}$

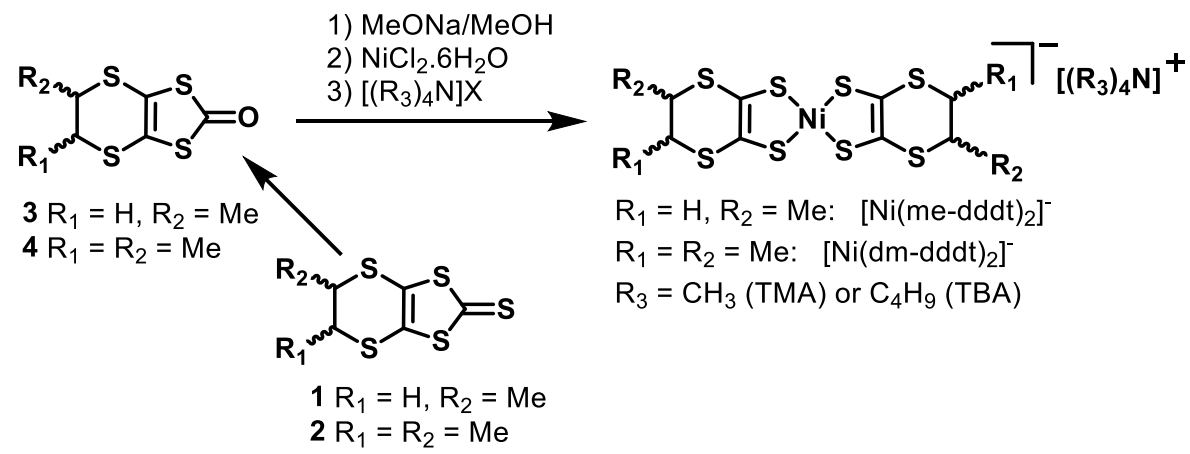

Scheme 3 Synthesis of chiral anionic Ni-bis(dithiolene) complexes. 
Recrystallization of the resulting powders in dichloromethane (DCM) afforded single crystals suitable for X-ray structure determination, which confirmed the absolute configuration of the complexes (vide infra). Then, electrocrystallization of the anionic complexes afforded, upon one-electron oxidation, the corresponding enantiopure and racemic neutral compounds [Ni(me-dddt $\left.)_{2}\right]_{\text {and }}\left[\mathbf{N i}(\mathbf{d m}-\mathbf{d d d t})_{2}\right]_{\text {as }}$ single crystals suitable for $\mathrm{X}$-ray structure determination and resistivity measurements.

\subsection{Crystal structures of the series (TBA)[Ni(me-dddt $\left.)_{2}\right]$}

The structures of the series of complexes (TBA)[Ni(me-dddt) ${ }_{2}$ ] (Fig. 3) are comparable to those of the series of (TBA) $\left[\mathbf{N i}(\mathbf{d m}-\mathbf{d d d t})_{2}\right]{ }^{40}$ Note however that we have now prepared and crystallized the racemic form as well, either by starting from the racemic precursor ( $\mathrm{rac}$ )-3 or by mixing stoichiometric amounts of enantiopure complexes (TBA)[Ni(S-me-dddt) $)_{2}$ and (TBA)[Ni(R-me-dddt $)_{2}$ ] in a scrambling experiment. Thus, we clearly demonstrate here that these complexes are flexible in solution. In both cases the same crystal structure was obtained, which is (TBA)[Ni(S-me-dddt)(R-me-dddt)], thus clearly demonstrating that ligand exchange occurs in solution. The enantiomerically pure compounds (TBA)[Ni(S-me-dddt $)_{2}$ ] and (TBA)[Ni(R-me-dddt $)_{2}$ ] crystallized in the non-centrosymmetric space group $P 1$ with two independent anionic complexes and two TBA cations in their asymmetric unit, while the racemic compound (TBA) [Ni(S-me-dddt)( $R$-me-dddt)] crystallized in the $P$ - 1 space group with two half metal complexes and one TBA cation in the asymmetric unit (Table S2, Fig. S50, ESI). In the latter, the two independent $\mathrm{Ni}$ ions are located on inversion centres, therefore all complexes present the $S, R$ configuration. However, for one of the two metal complexes, the ethylene group is equally distributed over two positions. This structural disorder is due to the alternation of [Ni(S-me-dddt)(R-me-dddt)] and $[\mathrm{Ni}(R$-me-dddt)(S-me-dddt)] complexes along the $a$ axis. 

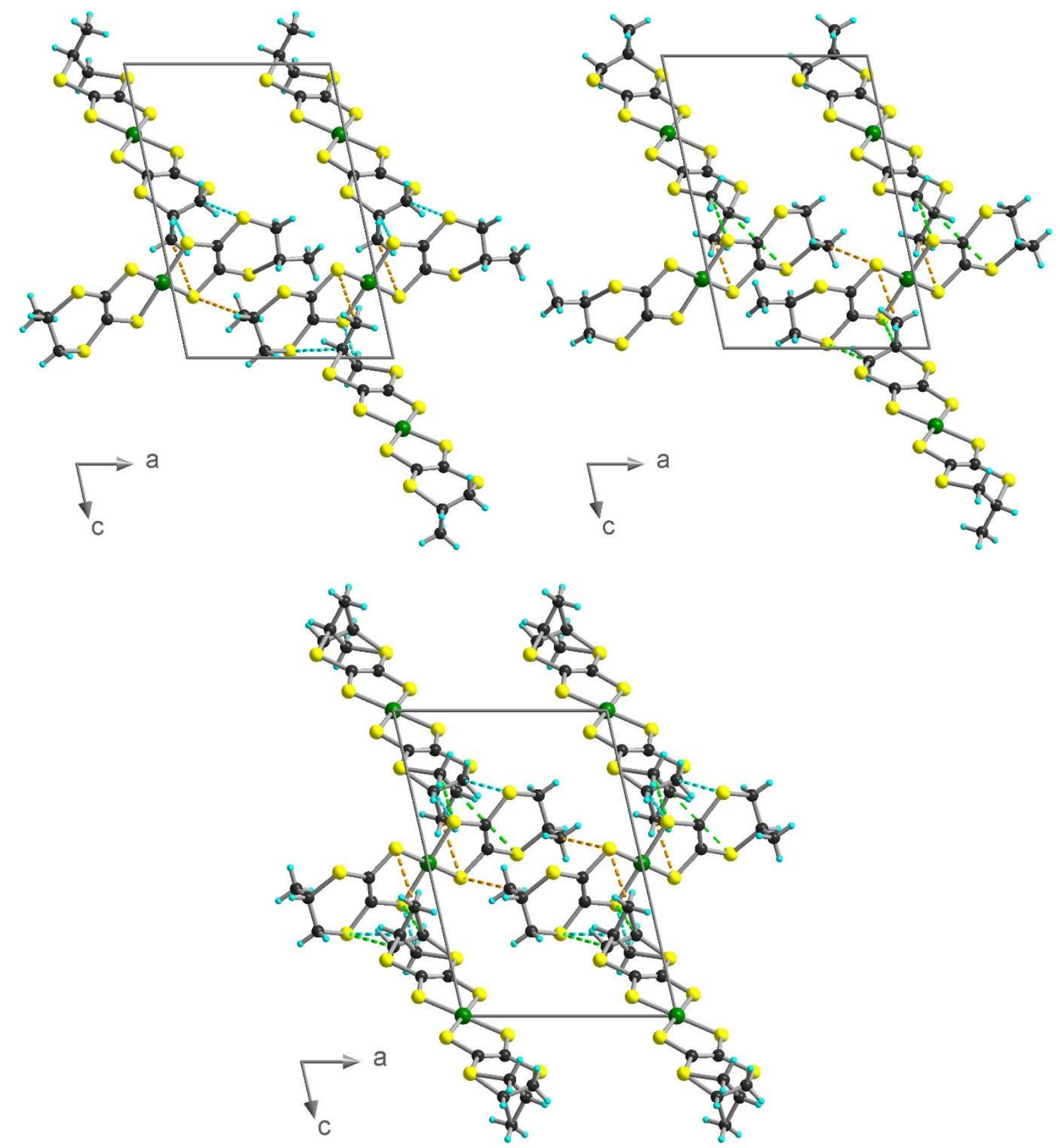

Fig. 3 Intermolecular $\mathrm{C}-\mathrm{H} \cdots \mathrm{S}$ interactions (dashed lines) between the anionic complexes in (TBA)[Ni(S-medddt) ${ }_{2}$ ], (TBA)[Ni(R-me-dddt) ${ }_{2}$ ] and (TBA)[Ni(S-me-dddt)(R-me-dddt)]. TBA cations are omitted for clarity. Color code: $\mathrm{C}$ (black), $\mathrm{H}$ (cyan), $\mathrm{S}$ (yellow), $\mathrm{Ni}$ (green). Cyan and light green dashed bonds correspond to the interactions involving $S$ and $R$ carbon atoms respectively, orange dashed bonds correspond to the methyl...S interactions which are present in both compounds.

Due to the presence of only one methyl substituent on the dithiolene ligand, both diastereoisomers cis and trans can, in principle, form. Here the enantiomeric composition of the anionic complexes, i.e. enantiopure versus racemic, strongly influences both the trans/cis nature of the diastereoisomers and the axial/equatorial arrangement of the methyl substituents in the solid state structures (Table 2 and Fig. S51, ESI). In the enantiomerically pure compounds (TBA)[Ni(S-me-dddt) ${ }_{2}$ ] and (TBA)[Ni(R-me$\mathbf{d d d t})_{2}$ ], both diastereoisomers cis and trans are observed. On the contrary, in (TBA)[Ni(S-me-dddt)( $R$ me-dddt)], the position of the $\mathrm{Ni}$ atoms on inversion centres imposes the trans isomer for both 
independent complexes. Similar axial (ax) and equatorial (eq) arrangement of the methyl substituents was previously observed in the crystal structure of enantiopure (TBA)[Ni(dm-dddt) ${ }_{2}{ }^{40}$ As a comparison, the all-eq conformation is largely more often encountered in the solid state structures of tetramethyl-bis(ethylenedithio)-tetrathiafulvalene (TM-BEDT-TTF) based radical cation salts, although mixed ax-eq or all-ax conformations occasionally occurred as well. ${ }^{49,50}$ Here we observe that the ax or eq conformation can be also influenced by the racemic or enantiopure configuration of the complexes, yet they are isostructural. Accordingly, in (TBA)[Ni(S-me-dddt) ${ }_{2}$ ] and (TBA)[Ni(R-me-dddt) ${ }_{2}$ ], the cis isomer presents the all-ax conformation and the trans isomer the ax-eq one, while in the racemic complex, with both independent molecules as trans isomers, the all-ax conformation is observed. This has an influence on the number of intermolecular contacts between metal complexes, which is more important in the racemic compound (Fig. S51, ESI).

Table 2 Structural configuration of anionic Ni complexes in the series of (TBA)[Ni(me-dddt) 2 ]

\begin{tabular}{|c|c|c|c|c|}
\hline \multirow{2}{*}{ Compound } & \multicolumn{2}{|c|}{ Complex 1 } & \multicolumn{2}{c|}{ Complex 2 } \\
\cline { 2 - 5 } & Diastereoisomer & $\mathrm{CH}_{3}$ positions & Diastereoisomer & $\mathrm{CH}_{3}$ positions \\
\hline (TBA)[Ni(S-me-dddt)2] & trans & ax-eq & cis & ax-ax \\
\hline (TBA)[Ni(R-me-dddt) $\left.)_{2}\right]$ & trans & eq-ax & cis & ax-ax \\
\hline (TBA)[Ni(rac-me-dddt) $]$ & trans & ax-ax & trans & ax-ax \\
\hline
\end{tabular}

For comparison purpose we have also prepared and crystallized the racemic (TBA)[Ni(S,S-dmdddt)( $\boldsymbol{R}, \boldsymbol{R}$-dm-dddt)] complex, both by using the racemic precursor ( $\mathrm{rac})-\mathbf{4}$ and by scrambling from equimolar (TBA)[Ni(S,S-dm-dddt) $\left.{ }_{2}\right]$ and (TBA)[Ni(R, $R$-dm-dddt) $)_{2}$ (Table S3). While the compound is isostructural with its enantiopure counterparts, ${ }^{40}$ the same conformational issues as in (TBA) [Ni(S-medddt)(R-me-dddt)] were not observed, the ax/eq orientation of the methyl substituents being the same as in the related enantiopure compounds (Fig. S52).

\subsection{Structure of the series (TMA)[Ni(dm-dddt) 2$]$}

The complexes of this series crystallized in the monoclinic system (Table S4). The enantiomerically pure compounds (TMA)[Ni(S,S-dm-dddt $\left.)_{2}\right]$ and (TMA)[Ni(R,R-dm-dddt $\left.)_{2}\right]$ crystallized in the noncentrosymmetric 12 space group with one independent $\mathrm{Ni}$ complex and two half cations in the asymmetric unit, while the racemic compound crystallized in the centrosymmetric 12/a space group, with two half complexes and two half cations in the asymmetric unit (Fig. S53-S54, ESI). In the latter, the ethylene groups are disordered and equally distributed over two positions. Moreover, since the $\mathrm{Ni}$ atoms lie on inversion centres, all complexes must present the $S, S, R, R$ configuration, leading to the formula (TMA)[Ni(S,S-dm-dddt)(R,R-dm-dddt)]. In the three compounds, the crystal packing shows 1D stacking of the complexes, with numerous $\mathrm{C}-\mathrm{H} \cdots \mathrm{S}$ intermolecular interactions and partial intermolecular overlap of the complexes along the $c$ direction (Fig. 4). The C-H...S interactions are characterized by C...S distances in the range 3.756-3.952 Å for (TMA)[Ni(S,S-dm-dddt) 2 ], 3.755-3.954 $\AA ̊$ for (TMA)[Ni(R,R-dm-dddt) 2 ], and 3.852-3.941 $\AA$ for (TMA)[Ni(S,S-dm-dddt)(R,R-dm-dddt)]. 

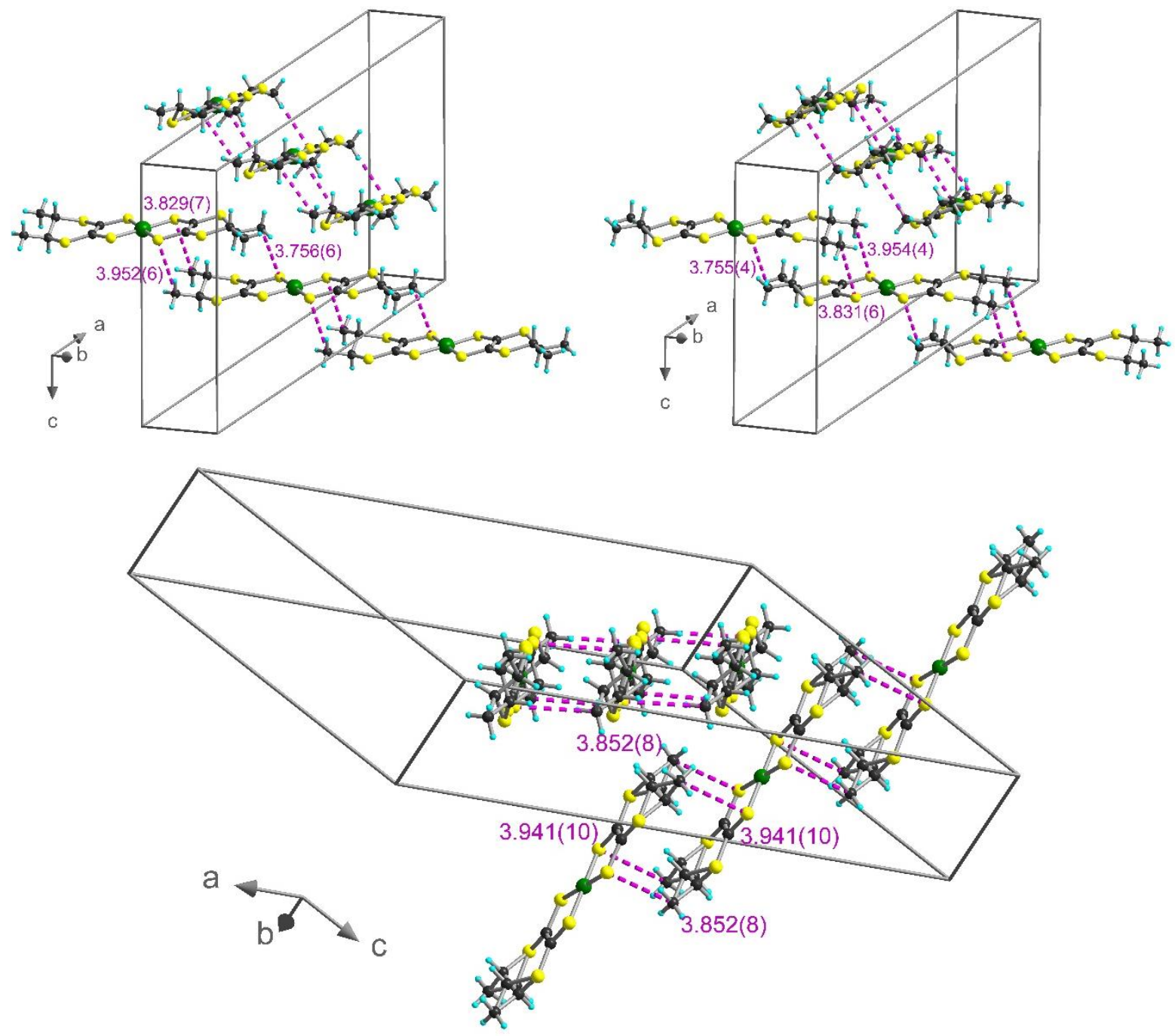

Fig. 4 Crystal packing of the anionic complexes in (TMA)[Ni(S,S-dm-dddt) 2 ], (TMA)[Ni(R,R-dm-dddt) 2 and (TMA)[Ni(S,S-dm-dddt)(R,R-dm-dddt)]. Intermolecular C-H $\cdots$ S interactions are represented in pink dashed lines. TMA cations are omitted for clarity.

In these three compounds the complexes present all-eq conformations, thus highlighting the paramount role of the cation when compared to the corresponding TBA series in which both all-ax and all-eq conformations are observed. ${ }^{40}$ This arrangement of the methyl substituents results in close contacts between complexes within the 1D chains, with a distance between two consecutive planes of complexes of $4.02 \AA$, for example, in (TMA)[Ni(S,S-dm-dddt)(R,R-dm-dddt)] (Fig. S55, ESI). Intermolecular S $\cdots S$ interactions of $4.3-4.6 \AA$ are also observed between complexes belonging to different 1D chains (Fig. S56, ESI).

\subsection{Structure of the series [ $\left.\mathrm{Ni}(\mathrm{me}-\mathrm{dddt})_{2}\right]$}

In this first series of neutral compounds, the enantiopure [Ni(S-me-dddt $)_{2}$ ] and [Ni(R-me-dddt $)_{2}$ ] complexes crystallized in the monoclinic non-centrosymmetric space group $P 2_{1}$ with one independent complex in the asymmetric unit, while the racemic [Ni(S-me-dddt)( $R$-me-dddt)] form crystallized in the space group $P 2_{1} / \mathrm{c}$ with half independent complex (Table S5, Fig. S57, ESI). They all present the same crystal packing, consisting of 2D layers of metal complexes in the $b c$ plane (Fig. 5). Two kind of 
intermolecular interactions can be observed in all of them as highlighted for the racemate in Fig. 6 . Moreover, $\mathrm{C}-\mathrm{H} \cdots \mathrm{S}$ short contacts are observed in the plane of the $2 \mathrm{D}$ layer, with $\mathrm{C} \cdots \mathrm{S}$ distances in the range 3.682-3.912 ̊̊ for [Ni(S-me-dddt) $)_{2}$ ], 3.694-3.915 $\AA$ for [Ni(R-me-dddt $)_{2}$ ] and 3.749-3.858 $\AA$ for [Ni(S-me-dddt)(R-me-dddt)] (Fig. S58-S60, ESI). Along the $a$ axis, the "out-of-plane" interactions are

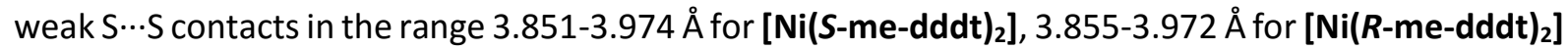
and 3.905-4.029 $\AA$ for [Ni(S-me-dddt)(R-me-dddt)] (Fig. S61, ESI). Contrary to the anionic racemic complex (TBA)[Ni(S-me-dddt)(R-me-dddt)] for which structural disorder was observed on the ethylene groups, in the neutral racemic complex no such disorder was present.

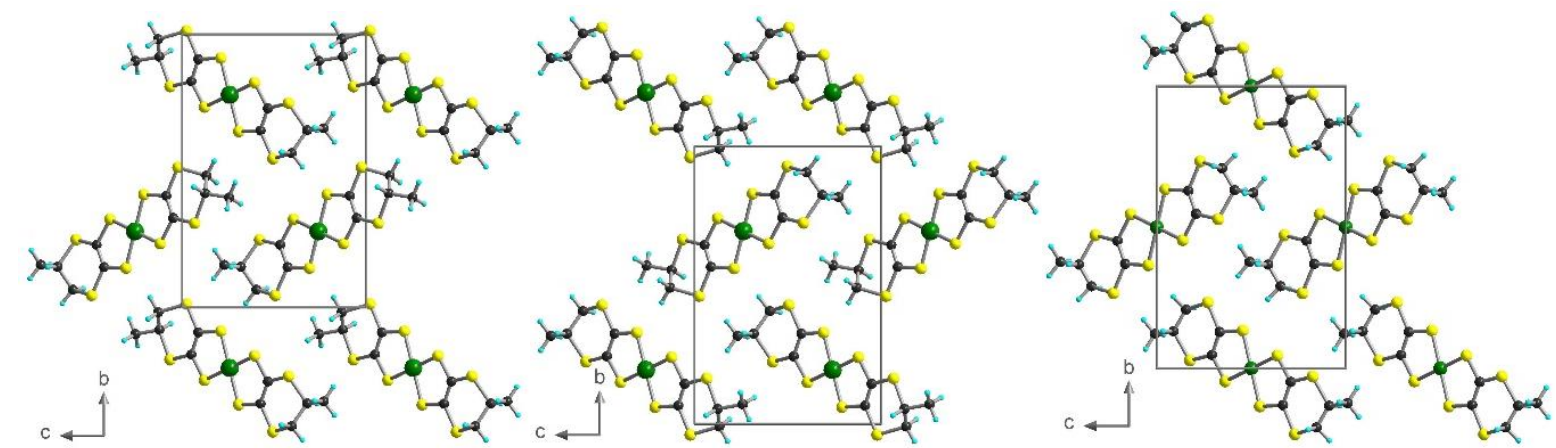

Fig. 5 Structures of [Ni(S-me-dddt $\left.)_{2}\right],\left[\mathrm{Ni}(\boldsymbol{R} \text {-me-dddt })_{2}\right]$ and $[\mathrm{Ni}(\mathrm{S}-\mathrm{me}-\mathbf{d d d t})(\boldsymbol{R}$-me-dddt $)]$ in the $b c$ plane. Color code: C (black), H (cyan), S (yellow), Ni (green).

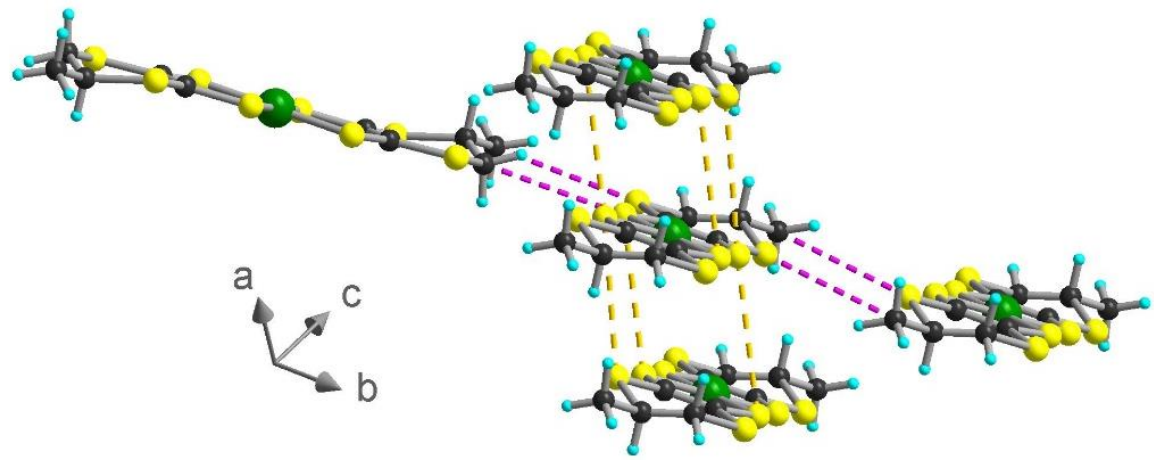

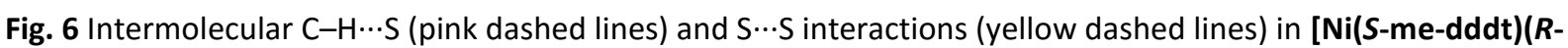
me-dddt)]. Color code: C (black), H (cyan), S (yellow), Ni (green).

\subsection{Structure of the series [ $\left.\mathrm{Ni}(\mathrm{dm}-\mathrm{dddt})_{2}\right]$}

The structure of $\left[\mathbf{N i}(\boldsymbol{R}, \boldsymbol{R}-\mathbf{d m}-\mathbf{d d d t})_{2}\right]$ was previously reported by us, ${ }^{40}$ therefore the related $(S, S)$ enantiomer and racemic compounds have now been prepared and structurally characterized. Complexes [Ni(S,S-dm-dddt $\left.)_{2}\right]$ and $\left[\mathbf{N i}(R, R-d m-d d d t)_{2}\right]$ crystallized in the triclinic space group $P 1$ with one independent complex in the asymmetric unit, while the racemic compound crystallized in the centrosymmetric space group $P-1$ with half of complex in the asymmetric unit (Table S6, Fig. S62, ESI). The methyl groups are located in equatorial positions. Unlike the enantiopure forms, the racemate shows structural disorder of the ethylene bridge, therefore both enantiomers are equally located on the same crystallographic site. The crystal packing shows numerous short intermolecular $\mathrm{S} \cdots \mathrm{S}$ distances between neighbouring complexes (Fig. 7 and S63, ESI). 

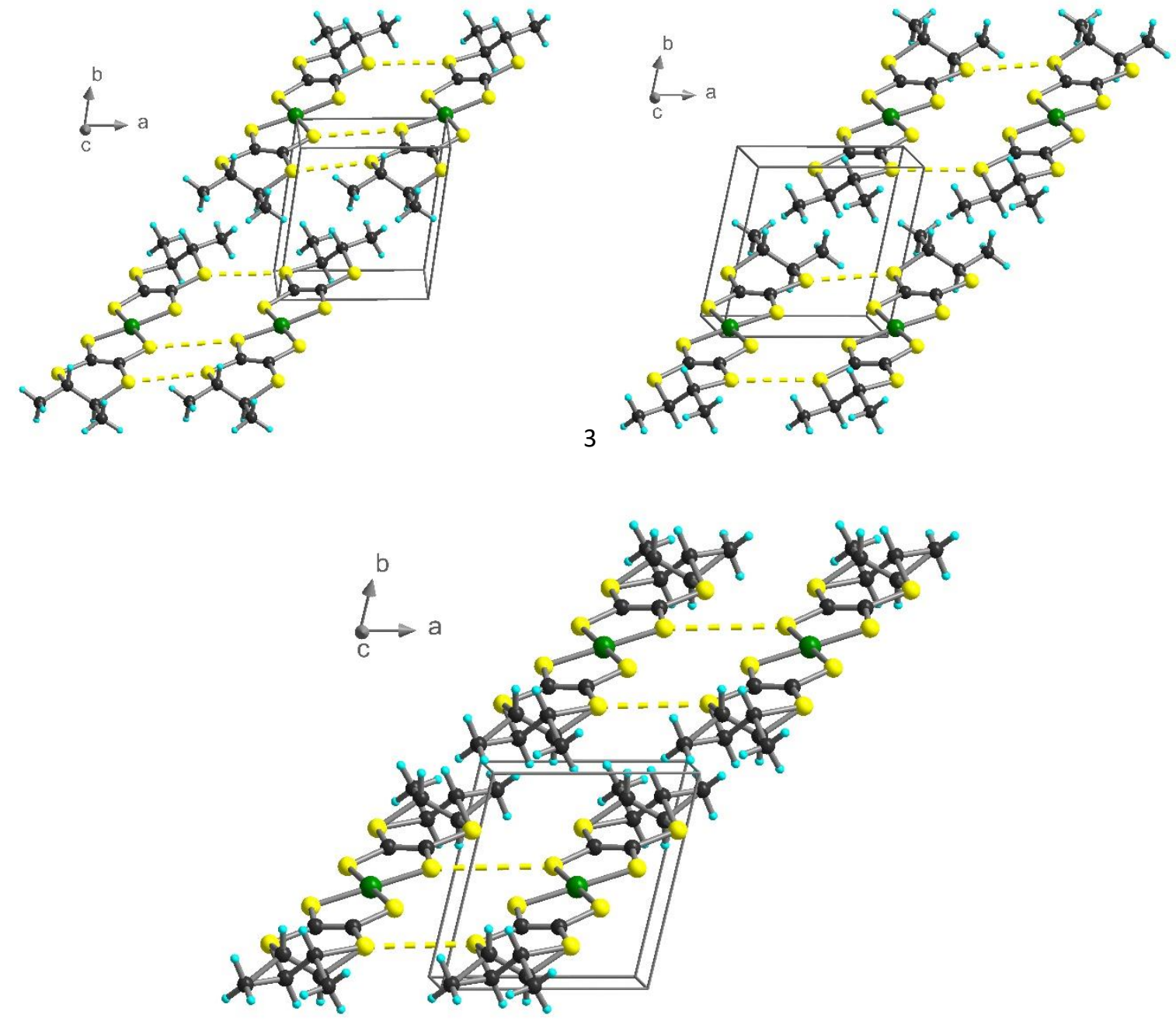

Fig. 7 Structures of [ $\left.\mathbf{N i}(S, S-d m-d d d t)_{2}\right],\left[\mathbf{N i}(\boldsymbol{R}, \boldsymbol{R}-\mathbf{d m}-\mathbf{d d d t})_{\mathbf{2}}\right]$ and $[\mathrm{Ni}(\boldsymbol{S}, \boldsymbol{S}-\mathbf{d m}-\mathbf{d d d t})(\boldsymbol{R}, \boldsymbol{R}$-dm-dddt)] in the $a b$ plane. Intermolecular S...S interactions of below $4 \AA$ are represented in yellow dashed lines. Color code: $\mathrm{C}$ (black), H (cyan), S (yellow), Ni (green).

\subsection{Single-crystal resistivity measurements}

\subsubsection{Single-crystal resistivity on TMA salts of anionic [Ni(dm-dddt $\left.)_{2}\right]$ complexes}

Single-crystal resistivity measurements on the TBA salts of anionic $\left[\mathrm{Ni}(\mathrm{S}, \mathrm{S}-\mathrm{dm}-\mathrm{dddt})_{2}\right]^{-}$and $[\mathrm{Ni}(R, R-\mathrm{dm}-$ $\left.\mathrm{dddt})_{2}\right]^{-}$complexes were previously performed. ${ }^{40}$ They showed semiconducting behaviour with a roomtemperature conductivity of about $10^{-6} \mathrm{~S} \mathrm{~cm}^{-1}$ and an activation energy of $380 \mathrm{meV}$ at ambient pressure. As discussed above, the change of the bulky TBA cation for the smaller TMA, induced a massive change in the packing of the (TMA)[Ni(dm-dddt $)_{2}$ ] series. Therefore, resistivity measurements have been performed first on the TMA salts of $\left[\mathrm{Ni}(\mathrm{dm} \text {-dddt })_{2}\right]^{-}$complexes in both their enantiopure and racemic forms. The use of a diamond anvil cell allowed us to measure the resistivity under pressures as high as 10.6 GPa (Fig. 8 and Table 3).

Although the TMA salts of this series show semiconducting behaviour as their TBA counterparts, the room temperature conductivity values at ambient pressure $\left(\approx 2-510^{-5} \mathrm{~S} \mathrm{~cm}^{-1}\right)$ are more than one order 
of magnitude higher than in the latter, thus highlighting the influence of the counter-ion on the conducting properties. Not surprisingly, since they are isostructural, the enantiopure and racemic (TMA)[Ni(dm-dddt) $)_{2}$ ] salts show similar values of conductivity, temperature and pressure dependence, as well as pressure dependence of the activation energy (Fig. 8).

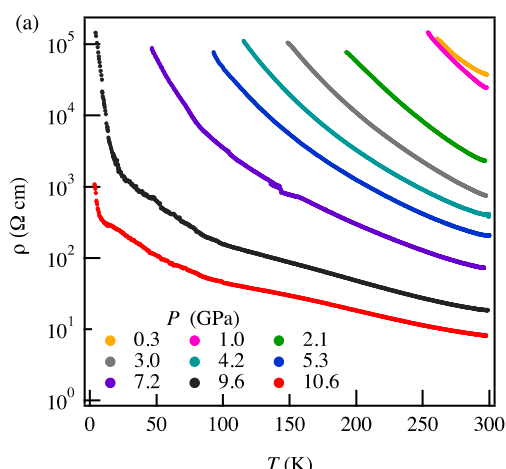

$T(\mathrm{~K})$

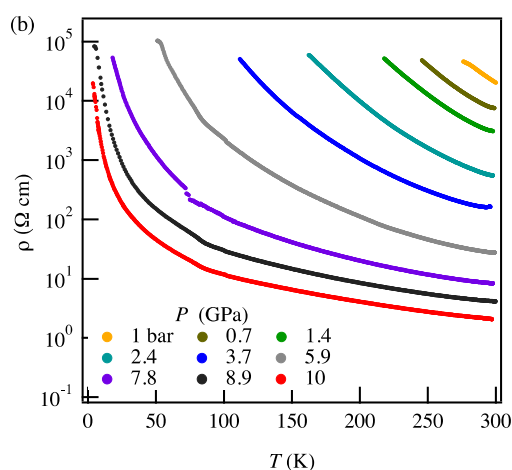

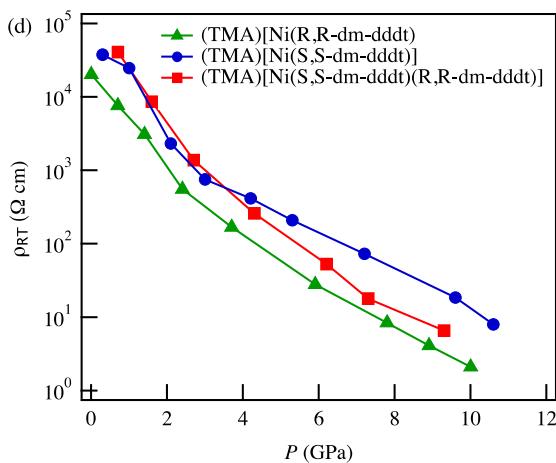

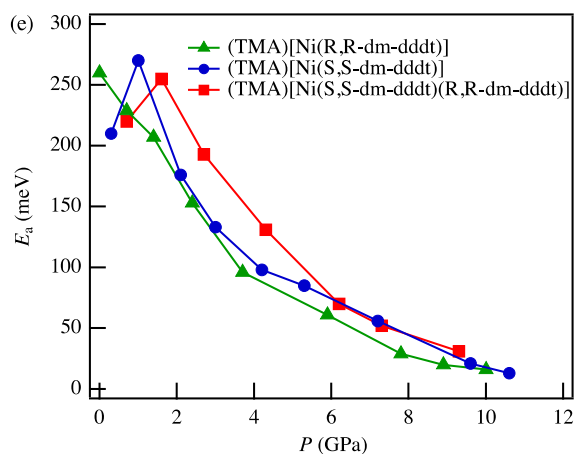

Fig. 8 Single-crystal resistivity measurements under various pressures for (a) (TMA)[Ni(S,S-dm-dddt) 2 ], (b) (TMA)[Ni(R,R-dm-dddt) $)_{2}$ and (c) (TMA)[Ni(S,S-dm-dddt)(R,R-dm-dddt)]. (d) Pressure dependence of room temperature resistivity. (e) Pressure dependence of activation energy.

Table 3 Room temperature conductivities and activation energies under various pressures for (TMA)[Ni(S,S-dmdddt)(R,R-dm-dddt)], (TMA)[Ni(S,S-dm-dddt)2] and (TMA)[Ni(R,R-dm-dddt) 2$]$

\begin{tabular}{|c|c|c|c|c|c|c|c|c|}
\hline \multicolumn{3}{|c|}{$\begin{array}{c}(\mathrm{TMA}) \\
{\left[\mathrm{Ni}(\mathrm{S}, \mathrm{S}-\mathrm{dm}-\mathrm{dddt})_{2}\right]}\end{array}$} & \multicolumn{3}{|c|}{$\begin{array}{c}(\mathrm{TMA}) \\
{\left[\mathrm{Ni}(R, R-\mathrm{dm}-\mathrm{dddt})_{2}\right]}\end{array}$} & \multicolumn{3}{|c|}{$\begin{array}{c}(\mathrm{TMA}) \\
{\left[\mathrm{Ni}(\text { rac-dm-dddt })_{2}\right]}\end{array}$} \\
\hline $\begin{array}{c}\text { Pressure } \\
\text { (GPa) }\end{array}$ & $\begin{array}{c}\rho_{\mathrm{RT}} \\
(\Omega . \mathrm{cm})\end{array}$ & $\begin{array}{c}E_{a} \\
(\mathrm{meV})\end{array}$ & $\begin{array}{c}\text { Pressure } \\
\text { (GPa) }\end{array}$ & $\begin{array}{c}\rho_{\mathrm{RT}} \\
(\Omega . \mathrm{cm})\end{array}$ & $\begin{array}{c}E_{a} \\
(\mathrm{meV})\end{array}$ & $\begin{array}{l}\text { Pressure } \\
\text { (GPa) }\end{array}$ & $\begin{array}{c}\rho_{\mathrm{RT}} \\
(\Omega . \mathrm{cm})\end{array}$ & $\begin{array}{c}\mathrm{E}_{\mathrm{a}} \\
(\mathrm{meV})\end{array}$ \\
\hline 0.3 & 37673 & 210 & 1 bar & 20230 & 260 & & & \\
\hline 1 & 24692 & 270 & 0.7 & 7610 & 229 & 0.7 & 41019 & 220 \\
\hline 2.1 & 2328 & 176 & 1.4 & 3100 & 207 & 1.6 & 8612 & 255 \\
\hline 3 & 753 & 133 & 2.4 & 556 & 153 & 2.7 & 1383 & 193 \\
\hline 4.2 & 415 & 98 & 3.7 & 168 & 96 & 4.3 & 260 & 131 \\
\hline 5.3 & 209 & 85 & 5.9 & 28 & 61 & 6.2 & 53 & 70 \\
\hline 7.2 & 73 & 56 & 7.8 & 8.4 & 29 & 7.3 & 18 & 52 \\
\hline 9.6 & 18.5 & 21 & 8.9 & 4.1 & 20 & 9.3 & 6.6 & 31 \\
\hline 10.6 & 8 & 13 & 10 & 2.1 & 16 & & & \\
\hline
\end{tabular}


At pressures as high as $9.3-10.6 \mathrm{GPa}$ the materials are still semiconductors, yet their room temperature conductivity reach values of $0.1-0.5 \mathrm{~S} \mathrm{~cm}-1$ and very low activation energies of $13-31$ meV (Table 3).

\subsubsection{Single-crystal resistivity of neutral [Ni(me-dddt $)_{2}$ and [Ni(dm-dddt $\left.)_{2}\right]$}

As discussed in the Introduction, neutral Ni-bis(dithiolene) complexes, in spite of their closed shell electronic structure, can show a dramatic increase of the conductivity under pressure. In our previous study, ${ }^{40}$ the conducting properties of neutral [Ni(dm-dddt $)_{2}$ ] complexes were not investigated. Resistivity measurements up to $11.2 \mathrm{GPa}$ have been carried out here on single crystals of the first series of neutral compounds [Ni(S-me-dddt $)_{2}$ ], [Ni(R-me-dddt $)_{2}$ ] and [Ni(S-me-dddt)(R-me-dddt)], bearing only one methyl substituent on the ethylene bridge (Fig. 9). The three materials show semiconducting behaviour in the whole temperature and pressure range, with maximum room temperature conductivities $\sigma_{R T}$ of $0.05 \mathrm{~S} \mathrm{~cm}^{-1}\left(10.2 \mathrm{GPa}, \mathrm{E}_{\mathrm{a}}=84 \mathrm{meV}\right), 0.02 \mathrm{~S} \mathrm{~cm}^{-1}\left(11.2 \mathrm{GPa}, \mathrm{E}_{\mathrm{a}}=133 \mathrm{meV}\right)$ and 1.7 $\mathrm{S} \mathrm{cm}^{-1}(10.0 \mathrm{GPa}, \mathrm{Ea}=26 \mathrm{meV})$ for the $S, R$ and racemic forms, respectively (Table 4). It appears thus that the racemic compound is much more conducting than the enantiopure counterparts for pressures above $8 \mathrm{GPa}$.
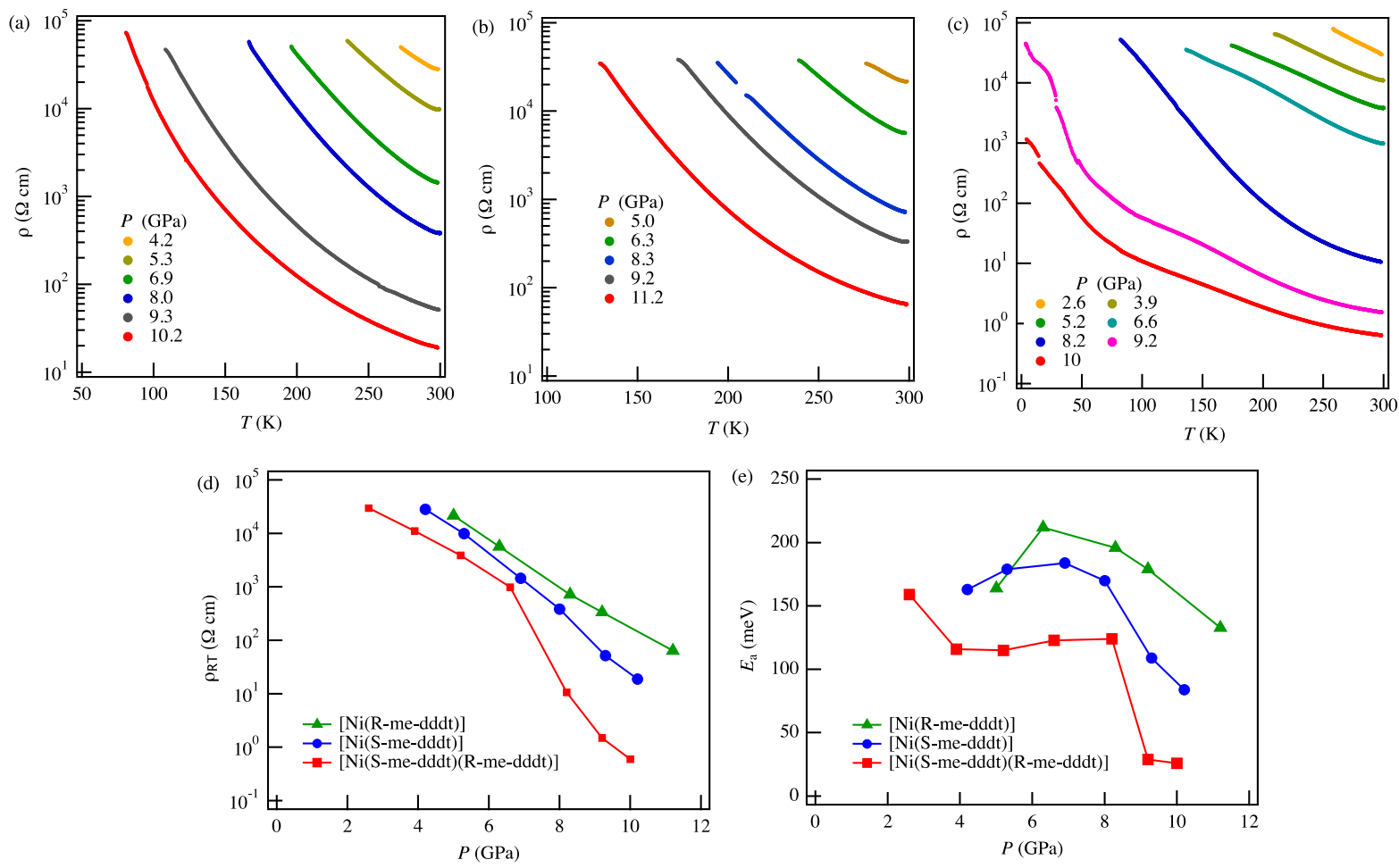

Fig. 9 Single-crystal resistivity measurements under various pressures for (a) [Ni(S-me-dddt) $\mathbf{2}_{\mathbf{2}}$,(b) [Ni(R-me$\mathbf{d d d t})_{2}$ ] and (c) [Ni(S-me-dddt)(R-me-dddt)]. (d) Pressure dependence of room temperature resistivity. (e) Pressure dependence of activation energy.

When looking at the pressure dependence of the room temperature conductivity (Fig. 9d) and of the activation energy (Fig. 9e) there is a clear discontinuity above $8 \mathrm{GPa}$ for the racemic compound, suggesting a phase transition impacting the electron transport properties. 
Table 4 Activation energies at different pressures and room-temperature conductivity under very high pressure for [Ni(S-me-dddt $\left.)_{2}\right],\left[\mathrm{Ni}(\boldsymbol{R} \text {-me-dddt })_{2}\right]$ and [Ni(S-me-dddt)(R-me-dddt)]

\begin{tabular}{|c|c|c|c|c|c|c|c|c|}
\hline \multicolumn{3}{|c|}{ [Ni(S-me-dddt $\left.)_{2}\right]$} & \multicolumn{3}{|c|}{ [Ni(R-me-dddt $\left.)_{2}\right]$} & \multicolumn{3}{|c|}{ [Ni(S-me-dddt)(R-me-dddt)] } \\
\hline $\begin{array}{c}\text { Pressure } \\
\text { (GPa) }\end{array}$ & $\begin{array}{c}\rho_{\mathrm{RT}} \\
(\Omega . \mathrm{cm})\end{array}$ & $\begin{array}{c}E_{a} \\
(\mathrm{meV})\end{array}$ & $\begin{array}{c}\text { Pressure } \\
\text { (GPa) }\end{array}$ & $\begin{array}{c}\rho_{\mathrm{RT}} \\
(\Omega . \mathrm{cm})\end{array}$ & $\begin{array}{c}E_{a} \\
(\mathrm{meV})\end{array}$ & $\begin{array}{c}\text { Pressure } \\
\text { (GPa) }\end{array}$ & $\begin{array}{c}\rho_{\mathrm{RT}} \\
(\Omega . \mathrm{cm})\end{array}$ & $\begin{array}{c}E_{a} \\
(\mathrm{meV})\end{array}$ \\
\hline & & & & & & 2.6 & 29754 & 159 \\
\hline 4.2 & 28233 & 163 & & & & 3.9 & 11132 & 116 \\
\hline 5.3 & 9915 & 179 & 5 & 21713 & 164 & 5.2 & 3868 & 115 \\
\hline 6.9 & 1446 & 184 & 6.3 & 5656 & 212 & 6.6 & 994 & 123 \\
\hline 8 & 386 & 170 & 8.3 & 721 & 196 & 8.2 & 10.6 & 124 \\
\hline 9.3 & 52 & 109 & 9.2 & 336 & 179 & 9.2 & 1.5 & 29 \\
\hline 10.2 & 19 & 84 & 11.2 & 64 & 133 & 10 & 0.6 & 26 \\
\hline
\end{tabular}

Remarkably, in the $\left[\mathbf{N i}(\mathbf{d m}-\mathbf{d d d} \mathbf{d})_{2}\right]$ series there is a clear difference between the enantiopure and racemic forms when considering the activation energy values, which are systematically higher for the latter (Fig. 10 and Table 5). This feature can be related to the presence of structural disorder in the racemate and not in the enantiopure salts (vide supra), inducing a better conductivity in the structurally ordered enantiopure materials. ${ }^{29,30,31}$
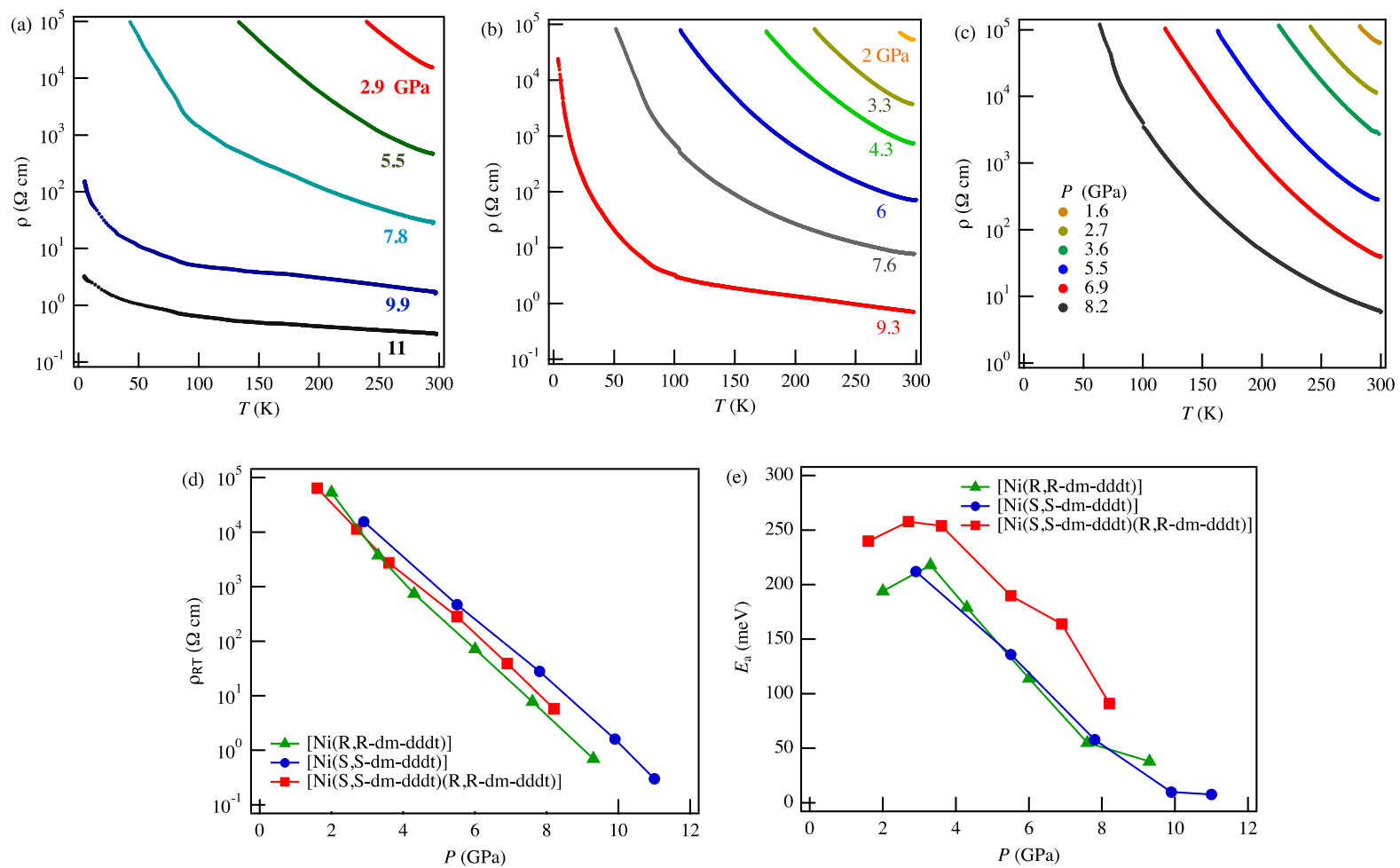
Fig. 10 Single-crystal resistivity measurements under various pressures for (a) [ $\mathbf{N i}(\mathbf{S}, \mathbf{S}$-dm-dddt) $\mathbf{2}$ ], (b) [Ni(R,R$\left.\mathbf{d m}-\mathbf{d d d t})_{2}\right]$ and (c) $[\mathrm{Ni}(S, S-d m-d d d t)(R, R-d m-d d d t)]$. (d) Pressure dependence of room temperature resistivity. (e) Pressure dependence of activation energy.

Conductivity values as high as $1.4-3.3 \mathrm{~S} \mathrm{~cm}^{-1}$ are reached for the enantiopure salts at the highest attempted pressures, yet the materials remain semiconducting. Interestingly, for equivalent pressures, the $\left[\mathbf{N i}(\mathbf{d m}-\mathbf{d d d} \mathbf{d})_{2}\right]$ salts are about one order of magnitude more conducting than the [Ni(me-dddt $\left.)_{2}\right]$ ones, very likely because of stronger intermolecular interactions and more favourable orbital overlaps (vide infra).

Table 5 Activation energies at different pressures and room-temperature conductivity under very high pressure for [Ni(S,S-dm-dddt $\left.)_{2}\right],\left[\mathrm{Ni}(\boldsymbol{R}, \boldsymbol{R}-\mathrm{dm}-\mathrm{dddt})_{2}\right]$ and [ $\left.\mathrm{Ni}(S, S-d m-d d d t)(R, R-d m-d d d t)\right]$

\begin{tabular}{|c|c|c|c|c|c|c|c|c|}
\hline \multicolumn{3}{|c|}{$\left[\mathrm{Ni}(S, S-\mathrm{dm}-\mathrm{dddt})_{2}\right]$} & \multicolumn{3}{|c|}{$\left[\mathrm{Ni}(R, R-\mathrm{dm}-\mathrm{dddt})_{2}\right]$} & \multicolumn{3}{|c|}{$[\mathrm{Ni}(S, S-\mathrm{dm}-\mathrm{dddt})(R, R-\mathrm{dm}-\mathrm{dddt})]$} \\
\hline $\begin{array}{c}\text { Pressure } \\
\text { (GPa) }\end{array}$ & $\begin{array}{c}\rho_{\mathrm{RT}} \\
(\Omega . \mathrm{cm})\end{array}$ & $\begin{array}{c}E_{a} \\
(m e V)\end{array}$ & $\begin{array}{c}\text { Pressure } \\
\text { (GPa) }\end{array}$ & $\begin{array}{c}\rho_{\mathrm{RT}} \\
(\Omega . \mathrm{cm})\end{array}$ & $\begin{array}{c}E_{a} \\
(\mathrm{meV})\end{array}$ & $\begin{array}{c}\text { Pressure } \\
\text { (GPa) }\end{array}$ & $\begin{array}{c}\rho_{\mathrm{RT}} \\
(\Omega . \mathrm{cm})\end{array}$ & $\begin{array}{c}E_{a} \\
(m e V)\end{array}$ \\
\hline & & & 2 & 53280 & 194 & 1.6 & 64231 & 240 \\
\hline 2.9 & 15625 & 212 & 3.3 & 3752 & 218 & 2.7 & 11453 & 258 \\
\hline 5.5 & 468 & 136 & 4.3 & 747 & 179 & 3.6 & 2748 & 254 \\
\hline 7.8 & 28 & 58 & 6 & 72 & 114 & 5.5 & 284 & 190 \\
\hline 9.9 & 1.6 & 9.8 & 7.6 & 7.8 & 55 & 6.9 & 39 & 164 \\
\hline 11 & 0.3 & 7.6 & 9.3 & 0.7 & 38 & 8.2 & 5.8 & 91 \\
\hline
\end{tabular}

Although the three series of salts are semiconductors, we have highlighted several striking differences between their conducting properties. First, the activated conductivity for the anionic and neutral series should have a different origin, related to their band filling (vide infra). Second, neutral dm-dddt complexes are more conducting than me-dddt ones, as a consequence of different packing and intermolecular orbital overlap triggered by the number of stereogenic centres on the ligands. Finally, within both families of neutral complexes, subtle differences are observed between the enantiopure and the racemic forms. Accordingly, in the $\left[\mathbf{N i}(\mathbf{m e - d d d t})_{2}\right]$ series the racemate shows a drastic increase of the conductivity at high pressures, while in the $\left[\mathbf{N i}\left(\mathbf{d m}-\mathbf{d d d} \mathbf{d}_{2}\right)_{2}\right.$ series the activation energies of the enantiopure salts are one order of magnitude smaller than those of the racemate, very likely because of the structural disorder observed in the latter. In order to shed light on the origin of the activated conductivity in our materials and also to rationalize the slightly higher conductivity of the [Ni(dm$\mathbf{d d d t})_{2}$ ] salts compared to the [Ni(me-dddt $)_{2}$ ] ones, first-principles density functional theory (DFT) and extended Hückel tight-binding band structure calculations have been performed.

\subsection{Band structure calculations}

In this section we first consider the electronic structure at ambient pressure of the two series of neutral complexes [ $\left.\mathbf{N i ( m e - d d d t})_{2}\right]$ and $\left[\mathbf{N i}(\mathbf{d m}-\mathbf{d d d} \mathbf{d})_{2}\right]$ and compare the results with those of the parent achiral system [ $\mathbf{N i ( d d d t )})_{2}$. Later we will consider the nature of the radical anion complexes in the two series of salts (TMA)[Ni(dm-dddt) ${ }_{2}$ ] and (TBA)[Ni(dm-dddt) $\left.{ }_{2}\right]$ at ambient pressure. Although we have described in detail the crystal structures of these solids in sections 2.3 to 2.6 we must now focus on the particular structural features which mostly determine the transport properties of these solids, i.e. the $\mathrm{S} \cdots \mathrm{S}$ interactions. 


\subsubsection{Neutral complexes}

The crystal structure of the [ $\left.\mathbf{N i}(\text { me-dddt })_{2}\right]_{\text {s }}$ series and the parent solid [ $\left.\mathbf{N i ( d d d t}\right)_{2}$ ] are clearly related. The two structures are shown in Fig. 11 where the $b c$ layers described in section 2.5 (but ac for $\left[\mathbf{N i}(\mathbf{d d d t})_{2}\right]^{19}$ ) are emphasized. If we consider the S $\cdots$. S contacts shorter than $4.0 \AA$ in the [Ni(me-dddt $)_{2}$ ] series, there are four different types of complex $\cdots$ complex interactions which are shown with different colours in Fig. 11a: (i) interactions along the stacking axis, i.e. the a-direction (interaction I), (ii) interactions between complexes in different stacks along the $c$-direction (interaction II) thus leading to ac layers, and two different types of interactions between stacks in the $b$-direction (interactions III and IV) thus interconnecting the ac layers and building a 3D lattice of $S \cdots S$ contacts. The $S \cdots S$ short contacts associated with each of the different interactions are reported in Table 6.

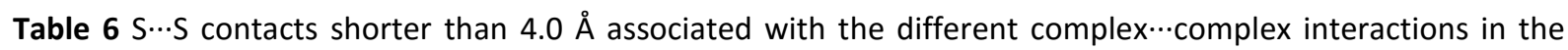
ambient pressure crystal structures of [Ni(dddt) $\left.{ }_{2}\right]$ and the [Ni(me-dddt) $\left.{ }_{2}\right]$ series, as labelled in Fig. 11 (values in Å)

\begin{tabular}{|c|c|c|c|c|}
\hline Interaction & {$\left[\mathrm{Ni}(\mathrm{dddt})_{2}\right]$} & {$\left[\mathrm{Ni}(S \text {-me-dddt })_{2}\right]$} & {$\left[\mathrm{Ni}(R \text {-me-dddt })_{2}\right]$} & $\begin{array}{c}\text { [Ni(S-me-dddt)(R-me- } \\
\text { dddt)] }\end{array}$ \\
\hline 1 & $\begin{array}{c}3.753(\times 2) \\
3.760(\times 2), 3.805(\times 2)\end{array}$ & $\begin{array}{c}3.850,3.902 \\
3.974\end{array}$ & $\begin{array}{c}3.855,3.913 \\
3.971\end{array}$ & $\begin{array}{c}3.905 \\
{[4.029(\times 2)]}\end{array}$ \\
\hline$I^{\prime}$ & $\begin{array}{c}3.844(\times 2) \\
3.904(\times 2), 3.953(\times 2)\end{array}$ & -- & -- & -- \\
\hline ॥ & 3.483 & 3.967 & 3.957 & 3.910 \\
\hline $\mathrm{II}^{\prime}$ & 3.590 & -- & -- & -- \\
\hline III & 3.684 & 3.988 & 3.984 & {$[4.008]$} \\
\hline IV & 3.837 & 3.902 & 3.891 & [4.099] \\
\hline $\mathrm{v}$ & 3.876 & -- & -- & -- \\
\hline
\end{tabular}

In the solid state of parent [ $\mathbf{N i ( d d d t )})_{2}$ ] there are two different types of stacks now running along $b$ (see Fig. 11b). ${ }^{19}$ As shown in Fig. 12, the overlap for both chains is of the very favourable head-to-tail type but whereas for chains 1 (interaction I in Fig. 11b) the three pairs of sulfur atoms are just on top of each other, for chains 2 (interaction I' in Fig. 11b) there is a noticeable sliding along the short molecular axis, leading to larger S...S distances and worse orbital overlap. As a matter of fact, the overlap in the chains of the [Ni(me-dddt $)_{2}$ ] series is of the same type but with a larger sliding so that in fact, only half of the $S$...S contacts survive. In other words the intra-chain interaction is progressively dismantled from chain 1 to chain 2 in [Ni(dddt) ${ }_{2}$ ] and then to the [Ni(me-dddt) ${ }_{2}$ ] series. This is clearly seen in the distances reported in Table 6 . There are of course other differences between the two structures. For instance, in [ $\mathbf{N i ( d d d t})_{2}$ ] there are two different types of inter-stacks interactions of the same type (II and II') but only one in the [Ni(me-dddt) ${ }_{2}$ ] series. In addition, interactions III and IV in the [Ni(me$\mathbf{d d d t})_{2}$ ] series are associated with two different complexes in one of the stacks but with only one in [Ni(dddt) ${ }_{2}$. 
a)

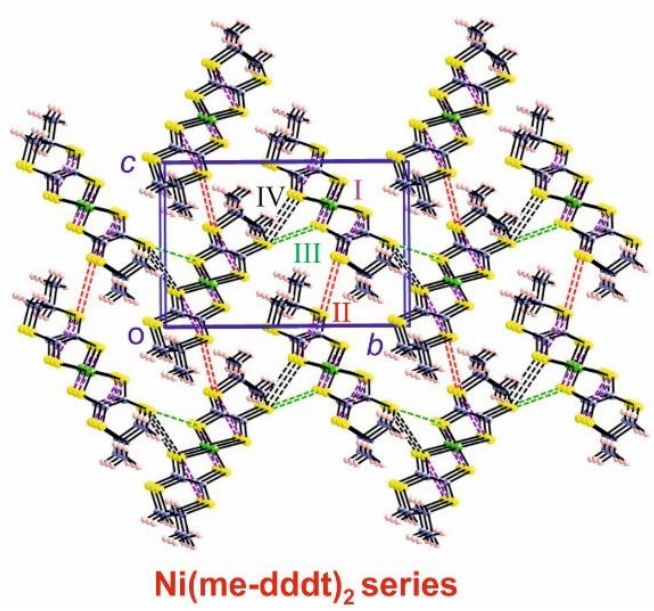

b)

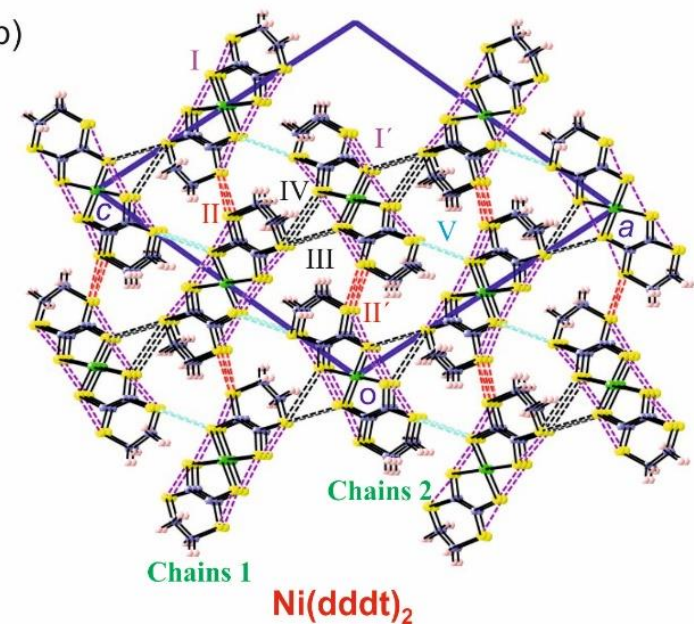

Fig. 11 View of the crystal structure along the direction of the Ni bis(dithiolene) stacks for the neutral [Ni(me$\mathbf{d d d t})_{2}$ ] series $(\mathrm{a})$ and $\left[\mathbf{N i}(\mathbf{d d d t})_{2}\right]^{19}$ (b). The different types of interaction are shown with different colours (see main text). Note that the direction of the stacks and the labelling of the axes are different in the two structures.

a)

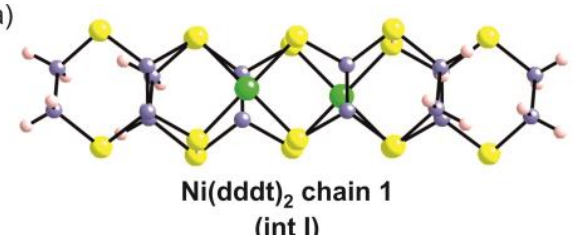

b)

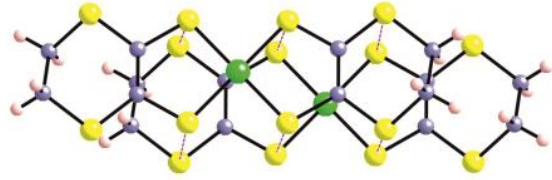

$\mathrm{Ni}(\text { dddt })_{2}$ chain 2

(int I')

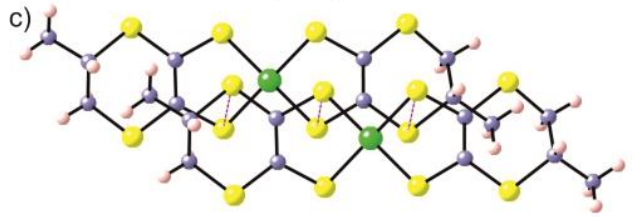

$\mathrm{Ni}(R \text {-me-dddt })_{2}$ chain

Fig. 12 Top view of the interaction along the stacks in [Ni(dddt) $\mathbf{2}_{2}(\mathrm{a})$ and $(\mathrm{b})$ as well as in the [Ni(me-dddt) $\mathbf{2}_{\mathbf{2}}$ series (c).

Although all of the S...S contacts are longer in the [Ni(me-dddt $)_{2}$ ] series, there are the intra-stack differences which mostly determine the variations in the band structure of the [ $\mathbf{N i ( d d d t )})_{2}$ and [ $\mathbf{N i}(\mathbf{m e}$ $\mathbf{d d d} \mathbf{t}_{\mathbf{2}}$ ] series of solids. The other interactions are associated with weaker electronic interactions which do not strongly differentiate among the structures. In contrast, the intra-stack interactions $\left(I\right.$ and $I^{\prime}$ in [Ni(dddt $)_{2}$ ] and $\mathrm{I}$ in the $[\mathbf{N i ( m e - d d d t})_{2}$ ] series) exhibit a clear trend which leads to an understanding of the calculated electronic structures (see Fig. 13a-b). According to extended Hückel calculations the absolute values of the calculated intermolecular interaction energies ${ }^{51,52}$ | $\beta_{\text {номо-номо }}$ and | $\beta_{\text {LUмо-LUмо }}$ for chain 1 of $\left[\mathbf{N i}(\mathbf{d d d t})_{2}\right.$ ] are $0.1054 \mathrm{eV}$ and $0.0377 \mathrm{eV}$, respectively, in agreement with the substantially smaller dispersion of the LUMO bands in Fig. 13a. The difference is due to the head-totail overlap and the different symmetry of the HOMO and LUMO with respect to the short molecular axis of the complex. The relative values of the $\left|\beta_{\text {номо-номо }}\right|$ for $\left[\mathbf{N i}(\mathbf{d d d t})_{\mathbf{2}}\right]_{\text {_chain }} \mathbf{1 /}\left[\mathbf{N i}(\mathbf{d d d t})_{\mathbf{2}}\right]_{\text {c chain }}$ 2 /average for the $\left[\mathbf{N i}(\mathbf{m e}-\mathbf{d d d t})_{2}\right]$ series are $1 / 0.1 / 0.06$. These values suggest that the HOMO-HOMO 
interactions are progressively dismantled from the $\left[\mathbf{N i}(\mathbf{d d d t})_{2}\right]$ chain 1 to $\left[\mathbf{N i}(\mathbf{d d d t})_{2}\right]$ chain 2 and the $\left[\mathbf{N i}(\text { me-dddt })_{2}\right]$ series. The relative values of $\left|\beta_{\text {Lumo-Lumo }}\right|$ are $1 / 0.9 / 0.06$, respectively. Taking into account the already smaller value of and $\left|\beta_{\text {Lumo-Lumo }}\right|$ for chain 1 , these values suggest that the sliding should influence in a lesser way the LUMO-LUMO interactions and particularly, those of the two chains in $\left[\mathrm{Ni}(\mathrm{dddt})_{2}\right]$.

a)

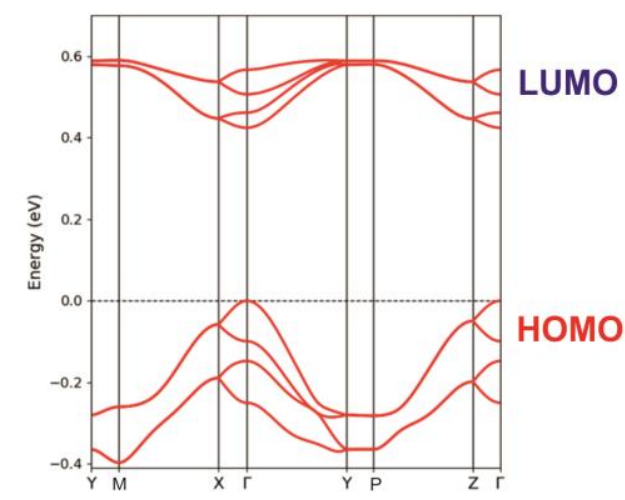

b)
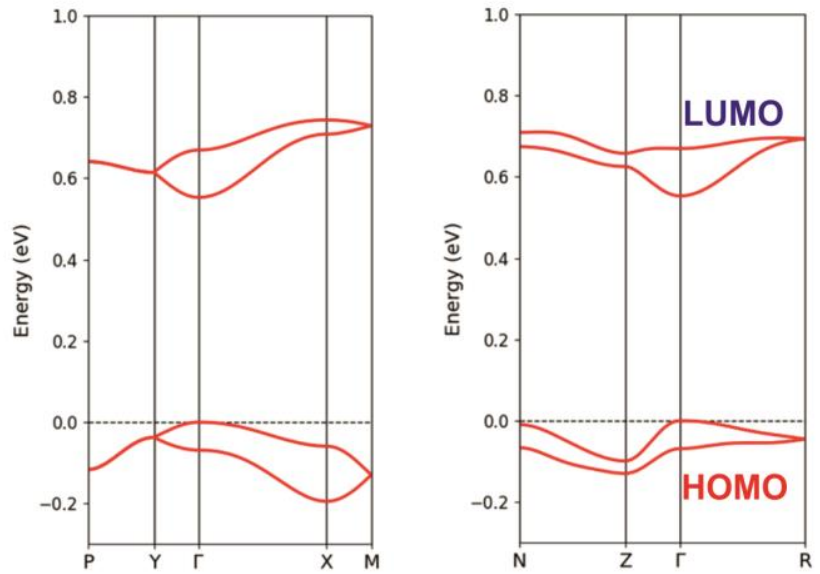

c)
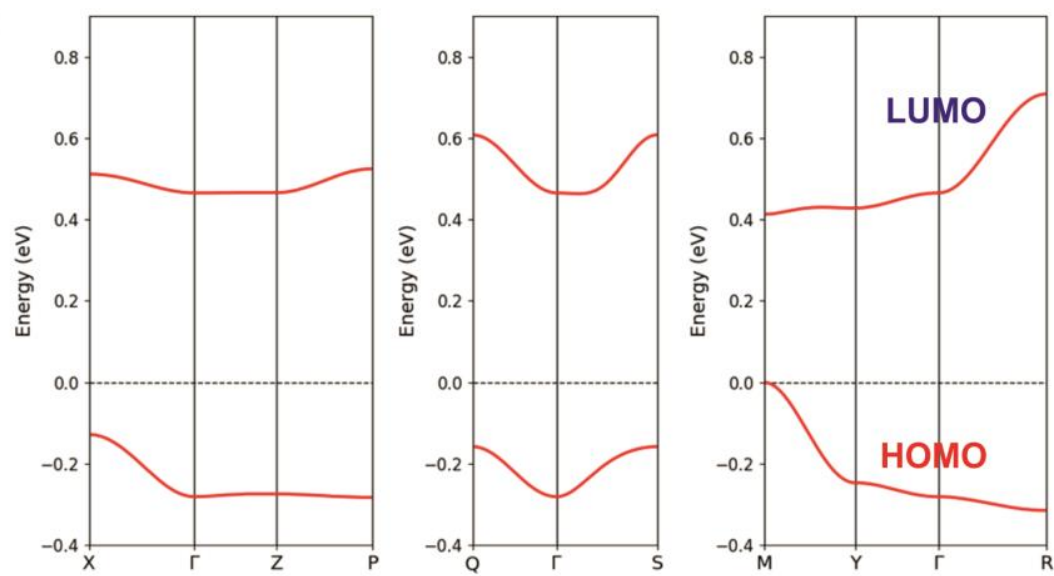

Fig. 13 DFT band structure for the neutral solids [Ni(dddt) $\mathbf{2}_{\mathbf{2}}$ (a) [Ni(R-me-dddt) $\mathbf{2}$ (b) and $\left[\mathbf{N i}(\boldsymbol{R}, \boldsymbol{R}-\mathbf{d m}-\mathbf{d d d t})_{2}\right](\mathrm{c})$. $\Gamma=(0,0,0), \mathrm{X}=\left(a^{*} / 2,0,0\right), \mathrm{Y}=\left(0, b^{*} / 2,0\right), \mathrm{Z}=\left(0,0, c^{*} / 2\right), \mathrm{M}=\left(a^{*} / 2, b^{*} / 2,0\right), \mathrm{P}=\left(0, b^{*} / 2, \mathrm{c}^{*} / 2\right), \mathrm{Q}=\left(a^{*} / 2,0, c^{*} / 2\right)$, $\mathrm{R}=\left(a^{*} / 2, b^{*} / 2, c^{*} / 2\right)$ and $\mathrm{S}=\left(-a^{*} / 2,0, c^{*} / 2\right)$. The dashed line refers to the highest occupied level. 
These qualitative considerations are clearly reflected in the calculated DFT band structures of Fig. 13ab. The dispersion of the HOMO bands is twice larger than that of the LUMO bands and the directions of strong dispersion are those parallel to the stacks direction (for instance $\Gamma-Y, M-X$ and $P-Z$ for [Ni(dddt $)_{2}$ ] but $\Gamma-\mathrm{X}$ for $\left.\left[\mathbf{N i}(\boldsymbol{R} \text {-me-dddt })_{2}\right]\right)$. Along both $\mathrm{M}-\mathrm{X}$ and $\mathrm{P}-\mathrm{Z}$ it is very clear that there is an avoided crossing of the HOMO bands: one of them, with larger dispersion, is mostly based on the orbitals of chain 1 whereas the other, with much smaller dispersion, is mostly based on the orbitals of chain 2 . Thus the top of the HOMO bands, which occurs at $\Gamma$ and will lead to hole carriers is dominated by the chain 1 . Such hole carriers have a smaller effective mass along the chains direction (i.e. strong dispersion around $\Gamma$ parallel to the chain direction) and a larger one (i.e. smaller dispersion) along the $(a+c)$ direction so that the hole carriers should behave as pseudo-1D carriers along the chains. A similar conclusion can be reached for the electron carriers (LUMO bands) - although now both types of chains contribute to the bottom of the LUMO bands - but since the effective mass is considerably larger because the interaction along the stacks is weaker, there are the holes that should dominate the conductivity. Thus, [ $\mathbf{N i ( d d d t )})_{2}$ ] at ambient pressure should be a semiconductor with direct band gap at $\Gamma$ and with a calculated activation energy of approximately $0.21 \mathrm{eV}$. It is clear from this analysis that the pressure dependence of the conductivity will strongly depend on the variation of the overlap within the chains as a function of pressure, with chains 1 playing the leading role. Conductivity data has not been reported for pressures lower than $5.9 \mathrm{GPa} .{ }^{19}$ The substantial decrease of the $b$ parameter up to this pressure and the reported activation energy at this pressure, $0.14 \mathrm{eV},{ }^{19}$ are consistent with a shortening of the intermolecular separation along the stacks without major changes in the overlap mode which, by increasing the band dispersion along the stacking directions, should decrease the activation energy and increase the carriers mobility.

The unit cell for the [Ni(me-dddt $)_{2}$ ] series contains only two complexes so that the band structures contain two HOMO and two LUMO bands. The band structure for [Ni(R-me-dddt $)_{2}$ ] is shown in Fig. 13b (those of [ $\mathbf{N i}\left(S\right.$-me-dddt) ${ }_{2}$ ] and [Ni(S-me-dddt)( $R$-me-dddt)] are very similar and are reported in Fig. S64, ESI, while the extended Hückel ones for all compounds are also very similar and are shown in Fig. S65, ESI). As expected, the dispersion of the $\mathrm{HOMO}$ bands is smaller than in [ $\mathbf{N i ( d d d t )}{ }_{2}$ ] thus leading to a larger activation energy which is calculated to be $0.275 \mathrm{eV}$ at ambient pressure $(0.279 \mathrm{eV}$ and $0.273 \mathrm{eV}$ for [Ni(S-me-dddt) $)_{2}$ ] and [Ni(S-me-dddt)(R-me-dddt)], respectively). The presence of the methyl substituent considerably perturbs the $\mathrm{C}-\mathrm{H} \cdots \mathrm{S}$ hydrogen bonding network at the region where the coupling between the above mentioned ac layers occurs (see Figs. 5-6 and S58-S60, ESI), ultimately leading to a substantial sliding of the complexes within the stacks (Fig. 12c) while keeping the general topology of the lattice. The solids of the [Ni(me-dddt) $)_{2}$ series are thus ambient pressure semiconductors with a direct gap at $\Gamma$ but, in contrast with [Ni(dddt) ${ }_{2}$ ], the contribution of electron and hole carriers to the conductivity should be more similar. The conductivity should be weaker and more 3D (i.e. the effective masses are heavier because the dispersion is smaller and less anisotropic around $\Gamma$ ) than in [ $\mathbf{N i ( d d d t )})_{2}$. When comparing these results with those of Table 4 one should be careful because of the absence of measurements at ambient pressure. There are three main intrinsic factors controlling the conductivity of a semiconductor: carrier mobility, activation energy and electron-phonon scattering, ${ }^{53}$ in addition to other extrinsic factors associated with defects, etc. When considering the values for similar pressures in Table 4 it is quite striking to see that the conductivity values for $\left[\mathbf{N i}(\boldsymbol{R} \text {-me-dddt })_{2}\right]$ are noticeably smaller than for the [Ni(S-me-dddt $\left.)_{2}\right]$ members of the series. Note also that while the activation energies for the latter are smaller for pressures around 6.9 GPa and higher, which is consistent with a somewhat higher dispersion and thus larger mobility, this 
is not the case at lower pressures. The difference between the values of the two enantiomeric forms does not seem to be justified neither on the basis of the electronic structure (mobility and activation energies) at ambient pressure nor on the expected closeness in electron-phonon scattering due to the structural similarity, thus suggesting that the differences should most likely be attributed to discrepancies in the hydrostatic pressure for different resistivity measurements or to extrinsic factors. Further progress along this direction completely depends on the possibility to obtain reliable structural data under pressure and the subsequent analysis by means of DFT calculations based on these structures. In any way, what may be firmly concluded is that introduction of the methyl substituent must decrease the conductivity and increase the activation energy at ambient pressure with respect to those of the parent [ $\mathbf{N i ( d d d t})_{2}$ ] solid as a result of the sliding induced along the short molecular axis of the complexes (see Fig. 12). Comparison of these results with those previously obtained for $\left[\mathbf{N i}(\mathbf{d d d t})_{2}\right]^{19}$ suggests that the methyl substitution should decrease the mobility and increase the activation energies at all pressures.

Bringing in a second methyl substituent completely changes the crystal structure which, as shown in

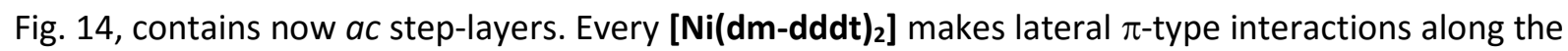
a-direction (interaction I in Fig. 14) leading to chains which are the steps of the step-layer. The [Ni(dmdddt $)_{2}$ ] units in one step are at the top of the hole between two [Ni(dm-dddt) $)_{2}$ ] units of the steps above and below (see Fig. 14b) so that there are two different inter-step interactions (interactions II and III). In that way every [ $\left.\mathbf{N i ( d m - d d d t})_{2}\right]_{\text {of }}$ one step is connected with four different [ $\left.\left.\mathbf{N i ( d m - d d d t}\right)_{2}\right]$ of adjacent steps and two of the same step. Finally, another relatively long interaction (interaction IV in Fig. 14a) provides a link between different step-layers. The S $\cdots . S$ contacts associated with these interactions are those reported in Table 7. It is clear that the S...S contacts are shorter in the [Ni(dmdddt $)_{2}$ ] series than in the [Ni(me-dddt $)_{2}$ ] one, thus suggesting stronger band dispersions. This can be clearly seen in the calculated band structure for [ $\mathbf{N i}(R, R-\mathbf{d m}-\mathbf{d d d t})_{2}$ ] shown in Fig. $13 \mathrm{c}$ (those for [ $\mathbf{N i}(S, S-$ $\mathbf{d m}$-dddt $\left.)_{2}\right]$ and $[\mathbf{N i}(S, S-d m-d d d t)(R, R-d m-d d d t)]$ are very similar and are reported in Fig. S66, ESI), where the total dispersion of the HOMO and LUMO bands is approximately $33 \%$ larger in the [Ni(dm$\mathbf{d d d t}_{2}$ ] salts. Nevertheless one must remind that the HOMO and LUMO are strongly directional so that looking solely to the $S \cdots S$ contacts can be sometimes misleading when assessing the strength of the intermolecular electronic interactions. This is why one needs to rely on the calculated intermolecular interaction energies ${ }^{51}$ associated with the HOMOs and LUMOs to have a hint on the strength of the

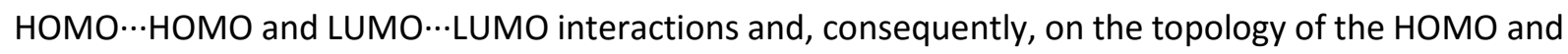
LUMO bands (dispersion, nature of the band gaps, etc.). For instance, in $\left[\mathbf{N i}(\boldsymbol{R}, \boldsymbol{R}-\mathbf{d m}-\mathbf{d d d t})_{2}\right]$ the absolute values of the calculated intermolecular interaction energies, $\left|\beta_{\text {номо-номо }}\right|$, are $0.0241 \mathrm{eV}$ (interaction I), $0.0360 \mathrm{eV}$ (interaction II), $0.0208 \mathrm{eV}$ (interaction III) and $0.0157 \mathrm{eV}$ (interaction IV). Those for the | $\beta_{\text {Luмо-Luмo }} \mid$ are $0.0188 \mathrm{eV}$ (interaction I), $0.0427 \mathrm{eV}$ (interaction II), $0.0205 \mathrm{eV}$ (interaction III) and $0.0108 \mathrm{eV}$ (interaction IV). The striking result is that the two interactions III and IV which are associated with long $\mathrm{S} \cdots \mathrm{S}$ contacts are not that different from those of the other interactions and, consequently, must play a substantial role in shaping the band structure. 


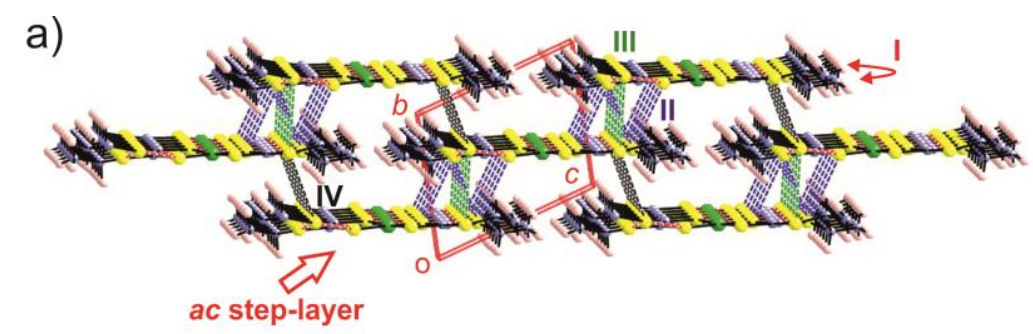

b)

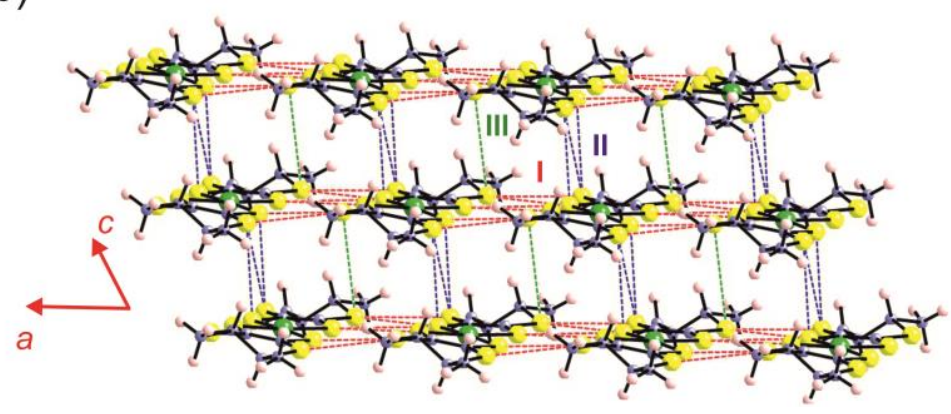

Fig. 14 Crystal structure (a) and one of the step-layers (b) of [Ni(S,S-dm-dddt) 2 , where the four different interactions (three within the step-layer, I to III, and one between step-layers, IV) are shown with different colors.

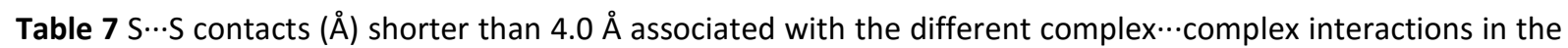
ambient pressure crystal structures of the [Ni(dm-dddt) 2 series as labelled in Fig. 14

\begin{tabular}{|c|c|c|c|}
\hline Interaction & {$\left[\mathrm{Ni}(S, S-\mathrm{dm}-\mathrm{dddt})_{2}\right]$} & {$\left[\mathrm{Ni}(R, R-\mathrm{dm}-\mathrm{dddt})_{2}\right]$} & $\begin{array}{c}\text { [Ni(S,S-me-dddt)(R, R-me- } \\
\text { dddt)] }\end{array}$ \\
\hline 1 & $\begin{array}{c}3.616,3.656,3.757,3.785 \\
3.965,3.977\end{array}$ & $\begin{array}{c}3.683,3.728,3.825,3.843 \\
{[4.035]}\end{array}$ & $\begin{array}{c}3.676(\times 2), 3.813(\times 2), \\
{[4.011]}\end{array}$ \\
\hline II & $3.728,3.825,3.839$ & $3.863,3.748,3.870$ & $3.645,3.759(\times 2)$ \\
\hline III & 3.935 & [4.011] & 3.951 \\
\hline IV & 3.996 & [4.087] & [4.113] \\
\hline
\end{tabular}

This observation has two important consequences. First, in contrast with naïve purely structural considerations, the inter-step-layer interactions (interaction IV) are important. Second, due to the above mentioned nature of the steps, with $\left[\mathbf{N i}(\mathbf{d m}-\mathbf{d d d t})_{2}\right]$ units in one step at the top of the hole between two [ $\mathbf{N i}(\mathbf{d m} \text {-dddt })_{2}$ ] units of the steps above and below, the effect of interactions II and III partially compensate in the HOMO bands, and together with some HOMO-LUMO interaction lead to practically nil dispersion in the $c$-direction. The only directions of the HOMO bands of the present steplayers exhibiting dispersion are those associated with the introduction of antibonding character along the $a$-direction (i.e., the $\Gamma \rightarrow \mathrm{X}, \Gamma \rightarrow \mathrm{Q}$ and $\Gamma \rightarrow \mathrm{S}$ directions in Fig. 13c). As suggested by these two observations, the top of the HOMO band is associated with the $\mathrm{M}$ point where there are strong antibonding interactions both along the $a$-direction of the step-layer and the inter-step-layer $b$ direction. Thus, the hole carriers should have small effective mass and contribute strongly to the conductivity. Third, the interplay between the three intra-layer interactions (I, II and III) changes in the LUMO bands because of the different (pseudo)symmetry of the HOMO and LUMO orbitals with respect to the short axis of the metal bis(dithiolene) unit. ${ }^{40}$ The only directions of the LUMO bands of the present step-layers exhibiting dispersion are those associated with the introduction of antibonding character along both the $a$ and $c$ directions (i.e., the $\Gamma \rightarrow R, \Gamma \rightarrow Q$ and $\Gamma \rightarrow S$ directions in Fig. 13c). As a 
result, the bottom of the LUMO band (i.e. the region with less LUMO …LUMO antibonding interactions) is associated with the $\mathrm{M}$ point, where the band is very flat, so that the electron carriers should have high effective mass and contribute weakly to the conductivity.

According to our study the three solids of the [Ni(dm-dddt) $)_{2}$ series are direct band gap semiconductors ( $M$ point) with the conductivity being dominated by the hole carriers. When compared with the solids of the [Ni(me-dddt) ${ }_{2}$ ] series, the stronger interactions lead to noticeably smaller activation energies $\left(0.206 \mathrm{eV}\right.$ for [ $\left.\mathbf{N i}(\boldsymbol{R}, \boldsymbol{R}-\mathbf{d m}-\mathbf{d d d t})_{2}\right], 0.199 \mathrm{eV}$ for [ $\left.\mathbf{N i}(S, S-d m-d d d t)_{2}\right]$ and $0.195 \mathrm{eV}$ for [Ni(S,S-dm-dddt)(R,R-dm-dddt)]). Assuming that no strong differences in the electronphonon scattering occur, such smaller activation energies and the lower effective hole masses are consistent with a better conductivity for the solids of the [Ni(S,S-dm-dddt $\left.)_{2}\right]$ series, as suggested by our measurements (vide supra). We do not find noticeable differences along the [Ni(dm-dddt) ${ }_{2}$ ] series. This is the first such comprehensive study on neutral nickel bis(dithiolene) complexes based on dddt and its methylated derivatives, highlighting the role of the number of stereogenic centres.

\subsubsection{Salts of anionic $\left[\mathrm{Ni}(\mathrm{dm}-\mathrm{dddt})_{2}\right]$ complexes}

The crystal structure of (TMA)[Ni(R,R-dm-dddt $)_{2}$ ] projected along the $b$-direction is shown in Fig. 15a. It can be described as a series of parallel $a b$ planes of $\mathrm{Ni}(R, R \text {-dm-dddt })_{2}$ complexes (see Fig. 16) in between which the TMA cations reside. In fact, every complex shown in the projection of Fig. 15a is a chain of complexes as shown in Fig. 15b. The shorter $S$...S contacts along the chains are 3.676, 3.907 and $4.004 \AA$ for (TMA) [Ni(R,R-dm-dddt $)_{2}$ ], 3.676, 3.911 and $4.005 \AA$ for (TMA)[Ni(S,S-dm-dddt $)_{2}$ ] and 3.659, 3.952 and $3.952 \AA$ for (TMA)[Ni(S,S-dm-dddt) $(R, R-d m-d d d t)]$. The unit cell contains two of these $a b$ planes (i.e four complexes).

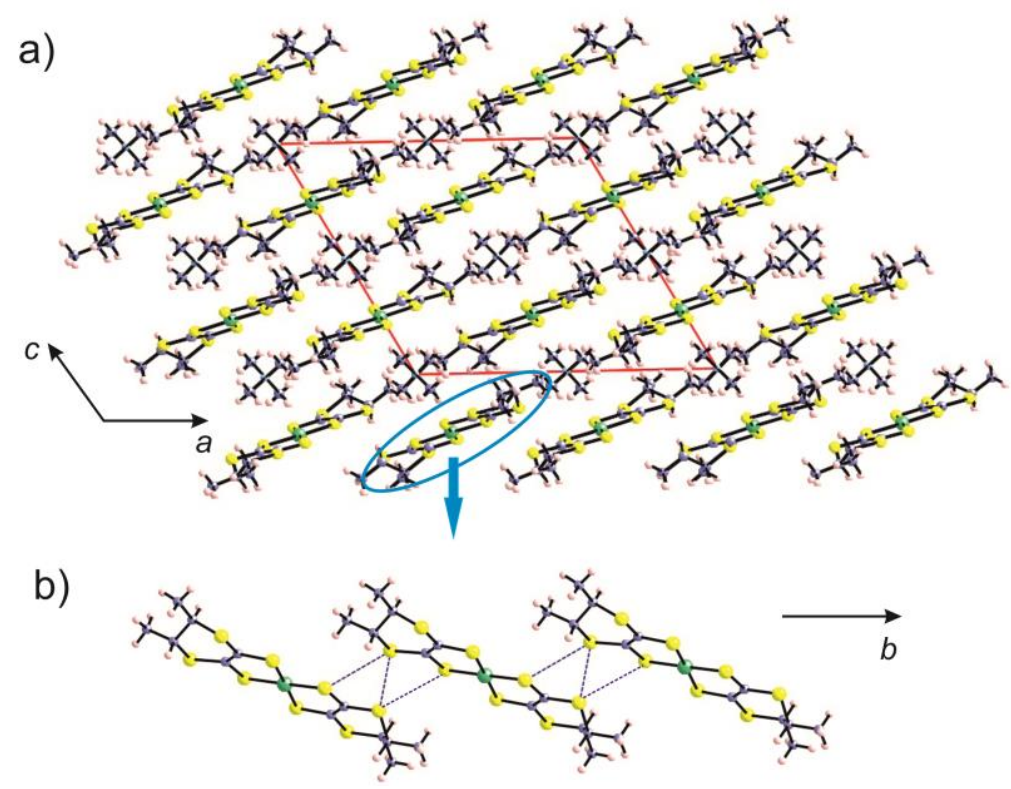

Fig. 15 Crystal structure of (TMA)[Ni(R,R-dm-dddt $)_{2}$ (a) with an emphasis on the $\mathrm{Ni}(R, R \text {-dm-dddt })_{2}$ chains along the $b$-direction (b). 


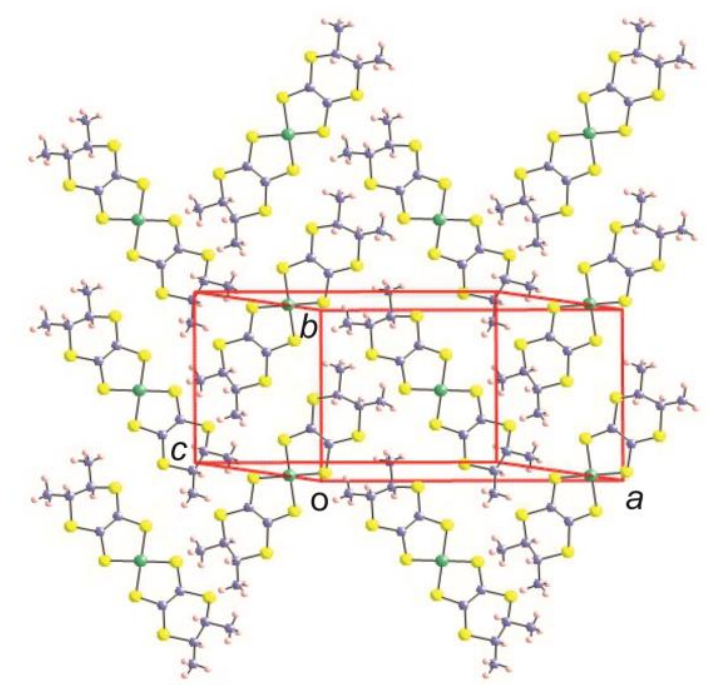

Fig. 16 View of one of the $a b$ planes in (TMA) [Ni( $\left.R, \boldsymbol{R}-\mathbf{d m}-\mathbf{d d d t})_{2}\right]$.

An important structural aspect of the system is that the successive $b$-chains of an $a b$ plane are oriented toward opposite directions with respect to the $b$-axis (see Fig.16) although this may be difficult to appreciate in the perspective of Fig. 15a. As a result, one could describe the structure as being built from double-chains along $b$ since one of these chains (for instance that highlighted in blue in Fig. 15) faces another chain with the same orientation in the next $a b$ plane. However, although such description may be structurally useful, one must take into account that the shortest $S \cdots S$ contacts between the two chains are $4.6-4.7 \AA$, while the shortest $S$...S contacts in between parallel chains of the $a b$ plane are within $4.3-4.7 \AA$. Thus, it is not expected that interactions other than those along the $b$-chains can really play a role in the electronic structure of the (TMA)[Ni(dm-dddt $\left.)_{2}\right]$ series.

It is worth recalling that for salts like those of the present series (with metal bis(dithiolene) radical anions) which are Mott semiconductors, the meaning of the activation energy is from the phenomenological viewpoint the same as for the band semiconductors discussed before. However, the origin of this activation energy is different since in a hopping mechanism the essential parameter is the electronic repulsion that must be overcome when the electron hopes from one site to the nearest neighbour (i.e. in the present case from a "SOMO" -singly occupied molecular orbital of the isolated radical anion, which is the equivalent of the LUMO for the neutral complexes- to a nearest neighbour SOMO). Although it is usually considered that it is the intra-site $U$ term (in this case $U_{\text {somo }}$ ) that determines the activation energy, the inter-site interactions can also affect this value even if in a lesser way. We have thus calculated the electronic structure of the (TMA)[Ni(R,R-dm-dddt) ${ }_{2}$ ] salt in different spin configurations: (i) ferromagnetic (FM), (ii) antiferromagnetic (AF) along the $b$-direction, and (iii) different ways to couple the AF $b$ chains within the $a c$ planes. The AF state along the chains with FM inter-chain coupling was found to be $0.7 \mathrm{~K} /$ complex more stable than the purely FM state. In fact all other states keeping the AF coupling along $b$ but different couplings in between chains lead to energies differing by less than $0.01 \mathrm{~K}$, which are beyond the precision of the present calculations. The stabilization provided by the $A F$ interactions along the chains were calculated to be $0.45 \mathrm{~K} /$ complex for (TMA)[Ni(S,S-dm-dddt) ${ }_{2}$ ] and $0.64 \mathrm{~K} /$ complex for (TMA)[Ni(S,S-dm-dddt)(R,R-dm-dddt)]. Thus we conclude that all salts of this series are weakly $\mathrm{AF}$ along the $b$-direction. 
a)

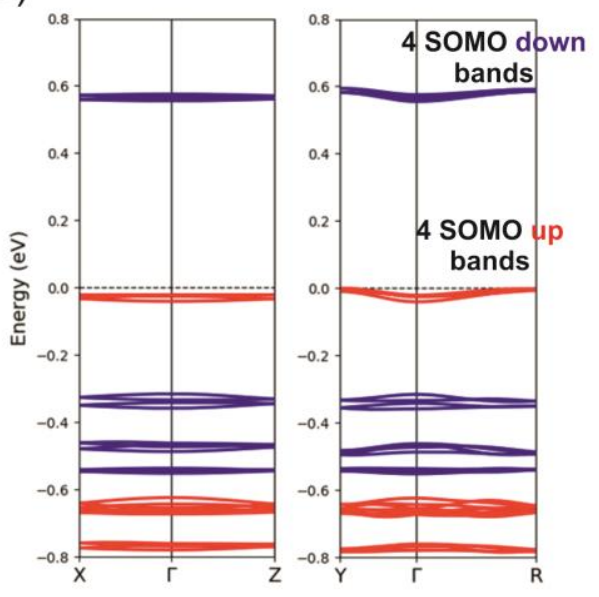

b)

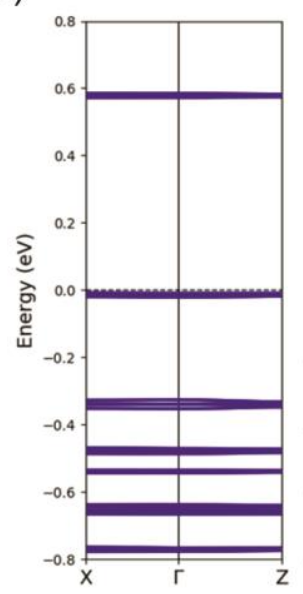

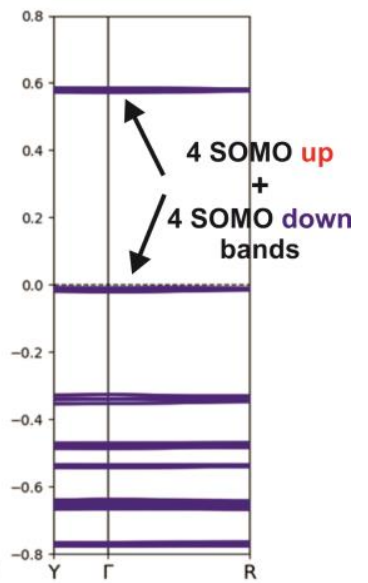

Fig. 17 DFT band structure for the ferromagnetic (FM) (a) and antiferromagnetic (AF) (b) states of (TMA)[Ni(R,R$\mathbf{d m}$-dddt $)_{2}$. Spin up and spin down bands are shown in red and blue, respectively. The FM state is calculated using the crystallographic cell $(a, b, c)$ containing 4 complexes and $\Gamma=(0,0,0), \mathrm{Y}=\left(0, b^{*} / 2,0\right), \mathrm{Z}=\left(0,0, c^{*} / 2\right), \mathrm{R}$ $=\left(a^{*} / 2, b^{*} / 2, c^{*} / 2\right)$ and $\mathrm{X}\left(a^{*} / 2,0,0\right)$. The AFM state is calculated using a double cell $\left(a^{\prime}=a, b^{\prime}=2 b\right.$ and $\left.c^{\prime}=c\right)$ containing 8 complexes and $\Gamma=(0,0,0), \mathrm{Y}=\left(0, b^{\prime *} / 2,0\right), \mathrm{Z}=\left(0,0, c^{\prime *} / 2\right), \mathrm{R}=\left(a^{\prime *} / 2, b^{\prime *} / 2, c^{\prime *} / 2\right)$ and $\mathrm{X}\left(a^{\prime *} / 2,0\right.$, $0)$. The dashed line refers to the highest occupied level. In (b) the spin up and spin down bands are identical although located in spatially different but equivalent sites so that only the blue bands are visible.

The calculated DFT spin-polarized band structure for both the FM and AF states are shown in Fig. 17. Since there are four complexes in the crystallographic cell of the FM state there are four SOMO up and four SOMO down bands rigidly shifted and with a very small dispersion (Fig. 17a). The double cell along $b$ of the AF state contains eight complexes. The band structure of Fig. 17b thus contains two groups of eight bands: four associated with spin up and four with spin down. The spin up and spin down bands are identical although located in spatially different but equivalent sites: this is why only the blue bands are visible. The estimated activation energies at ambient pressure are $0.277 \mathrm{eV}, 0.278 \mathrm{eV}$ and $0.289 \mathrm{eV}$ for (TMA)[Ni(R,R-dm-dddt) ${ }_{2}$ ], (TMA)[Ni(S,S-dm-dddt) $)_{2}$ and (TMA)[Ni(S,S-dm-dddt)(R,R-dm-dddt)], respectively. These values seem to be in quite good agreement with our measurements given the difficulties in both the theoretical and experimental determination. Note that the separation between the two sets of filled and empty SOMO bands is practically the same for the FM and AFM states which means that it is the $U_{\text {somo }}$ repulsion which determines the activation energy. Pressure should increase the hopping probability and thus increase the conductivity.

The last salt we have studied is (TBA)[Ni(S,S-dm-dddt)( $R, R$-dm-dddt)]. As shown in Fig. 18a, if the cations are ignored the crystal structure can be described as a series of parallel planes containing two different radical anions ( $\mathrm{A}$ and $\mathrm{B}$ ). An extended view of one of these planes having $a$ and $(b+c)$ as the two main directions is shown in Fig. 18b. The other salts of this series also exhibit two different complexes in the crystal structure, everyone associated with one of the chains along $a$. However it must be noticed that no $\mathrm{S} \cdots \mathrm{S}$ contacts shorter than $5.0 \AA$ occur in the structure so that the coupling between different complexes will essentially occur through the Coulomb field of the ions. The shorter S $\cdots \mathrm{S}$ contacts $(5.0-5.4 \AA)$ occur along the $a-(\cdots \mathrm{A} \cdots \mathrm{A} \cdots \mathrm{A} \cdots$ and $\cdots \mathrm{B} \cdots \mathrm{B} \cdots \mathrm{B} \cdots)$ and the $-a+(b+c)$-directions $(\cdots A \cdots B \cdots A \cdots B \cdots$, see Fig. 18b). We have calculated the electronic structure for (i) the FM state, (ii) several spin configurations with either FM and/or AF interactions along these directions, which can be generated with a double cell along $a$. The spin configuration with AF interactions along the $-a+(b+c)$ direction and FM along $a$ was found to be $0.84 \mathrm{~K} /$ complex more stable than the ferromagnetic state. 
This seems to be in good agreement with previous magnetic measurements for (TBA)[Ni(S,S-dmdddt $)_{2}$ ] which indicated the occurrence of isolated $S=1 / 2$ spin carriers which establish very weak antiferromagnetic interactions. ${ }^{40}$ Although the different spin configurations were found to be very similar in energy (i.e. within $0.45 \mathrm{~K} /$ complex) analysis of the results suggests that the $\mathrm{A} \cdots \mathrm{B}$ magnetic interactions dominate the magnetic coupling.

a)

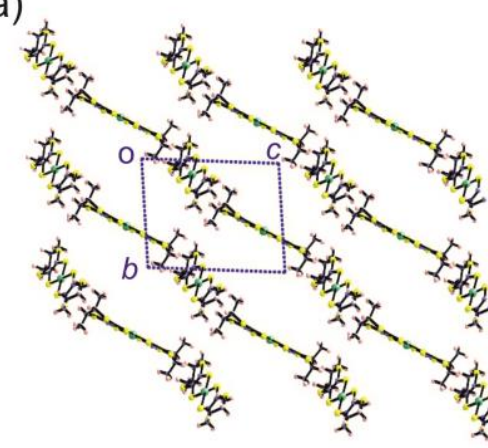

b)

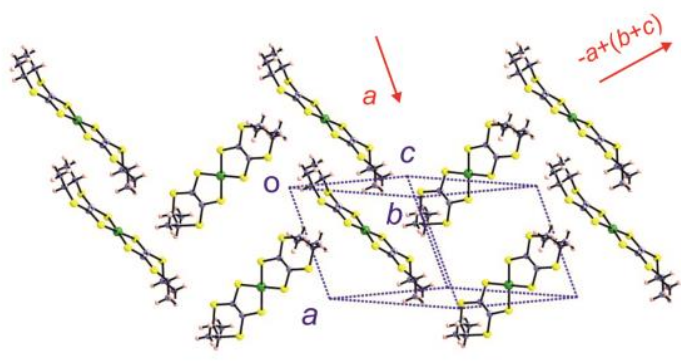

Layer

Fig. 18 (a) Crystal structure of (TBA)[Ni(S,S-dm-dddt)( $R, R$-dm-dddt)] where the cations have been removed. (b) View of one of the layers discussed in the text.

The calculated band structure for the FM and the AF state along $-a+(b+c)$ are shown in Fig. 19. Note that both states can be described with the crystallographic cell containing one molecule $A$ and one molecule B, so that there is one pair of SOMO up bands and one pair of SOMO down bands. Those associated with the $A$ and $B$ radical anions do not occur at the same energy because of the slight geometrical differences in the two radical anions. Again it is the $\mathrm{U}_{\text {sомo }}$ repulsion which determines the activation energy. The activation energy is estimated to be in the range $0.222-0.301 \mathrm{eV}$ which, as expected, is comparable to that of the (TMA)[Ni(dm-dddt) 2 ] series. In fact, the activation energy strongly depends on how much different are the two radical anions (i.e. it increases as the two radical anions become more similar) and consequently may be quite pressure dependent.

a)

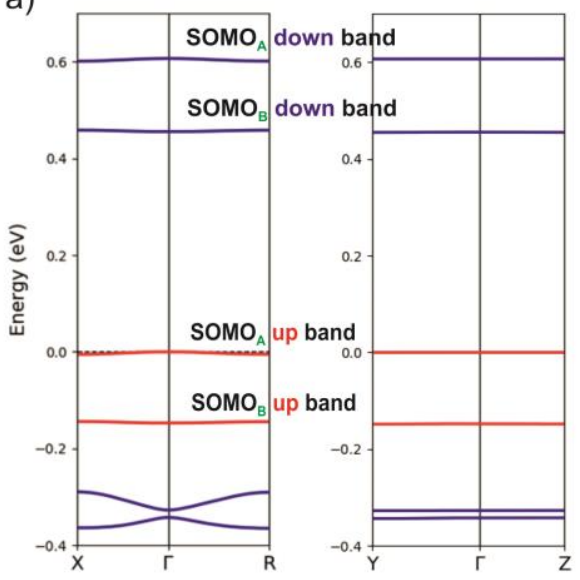

b)

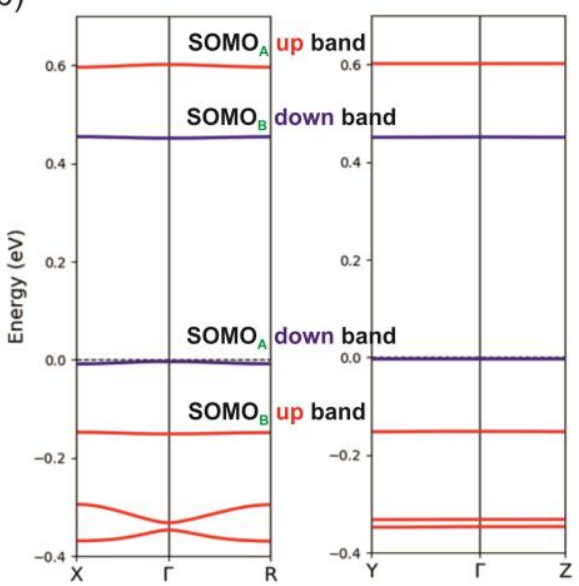

Fig. 19 DFT band structure for the ferromagnetic (FM) (a) and antiferromagnetic (AF) along $-a+(b+c)$ (b) states of (TBA)[Ni(S,S-dm-dddt)(R,R-dm-dddt)]. Spin up and spin down bands are shown in red and blue, respectively. Both states are calculated using the crystallographic cell $(a, b, c)$ containing two metal bis(dithiolenes) and $\Gamma=$ $(0,0,0), \mathrm{Y}=\left(0, b^{*} / 2,0\right), \mathrm{Z}=\left(0,0, c^{*} / 2\right), \mathrm{R}=\left(a^{*} / 2, b^{*} / 2, c^{*} / 2\right)$ and $\mathrm{X}\left(a^{*} / 2,0,0\right)$. The dashed line refers to the highest occupied level. 


\section{Conclusions}

Enantiopure dithiolene ligands containing one or two stereogenic centres and methyl susbtituents have been used to prepare complete series (both enantiomers and racemate) of monoanionic and neutral chiral nickel(II) bis(dithiolene) complexes. First, the thione precursors me-dddt (1) and dm-dddt (2), prepared as racemic mixtures and then isolated as pure enantiomers by chiral HPLC, have been structurally and chiroptically characterized. Experimental CD spectra corroborated with TD-DFT calculations indicate predominance of the equatorial conformer for $\mathbf{1}$ and of the bis-axial one for $\mathbf{2}$. The structural analysis of the monoanionic complexes show that the TBA salts of [Ni(me-dddt $)_{2}$ ] and $\left[\mathrm{Ni}(\mathrm{dm}-\mathrm{dddt})_{2}\right]$ are isostructural, with long $\mathrm{S} \cdots \mathrm{S}$ intermolecular distances, while changing the cation for the smaller TMA one, completely different structures are obtained, thus emphasizing the predominant role of the counter-ion over the number of methyl substituents. In each series, however, the enantiopure and racemic salts are isostructural and show semiconducting properties. The different packing between the TBA and TMA compounds results in one order of magnitude superior conductivity for the latter, that is $510^{-5}$ versus $10^{-6} \mathrm{~S} \mathrm{~cm}^{-1}$ at room temperature and ambient pressure. Interestingly, applying hydrostatic pressures in a diamond anvil cell allows to reach conductivities up to $510^{-1} \mathrm{~S} \mathrm{~cm}^{-}$ ${ }^{1}$ at around $10 \mathrm{GPa}$ for the (TMA)[Ni(dm-dddt $)_{2}$ ] series. DFT band structure calculations for these Mott insulators afford activation energy values in agreement with the experimental ones and bring strong evidence for the occurrence of antiferromagnetic ground states. The neutral closed-shell complexes [Ni(me-dddt $)_{2}$ ] and $\left[\mathbf{N i}(\mathbf{d m}-\mathbf{d d d t})_{2}\right.$ ] have been obtained by electrocrystallization of the anionic precursors. It is shown now that the number of stereogenic centres has a paramount importance since it strongly modulates the crystal structures. Indeed, while the crystal structures of the [Ni(me-dddt) ${ }_{2}$ ] series and that of the parent achiral [ $\mathbf{N i}(\mathbf{d d d t})_{2}$ ] are clearly related, consisting in stacks of complexes with a noticeable sliding along the short axis of the molecules in the former, bringing in a second methyl substituent in [ $\mathbf{N i}(\mathbf{d m}-\mathbf{d d d t})_{2}$ ] completely changes the crystal structure. As a consequence, although both series of chiral complexes are band gap semiconductors, the stronger intermolecular interactions in [ $\mathbf{N i}(\mathbf{d m}-\mathbf{d d d t})_{2}$ ] than in [ $\mathbf{N i}(\mathbf{m e - d d d t})_{2}$ ] lead to noticeably smaller activation energies, in agreement with the better conductivity measured for the former. This combined crystallographic and theoretical study remarkably correlates the structural changes, induced by the substitution of dddt with methyl groups in single component conductors based on nickel bis(dithiolene) complexes, with the fine modulation of the band structure directly related to the conducting properties. High pressure conductivity measurements afford a drastic increase of the conductivity values, while keeping the

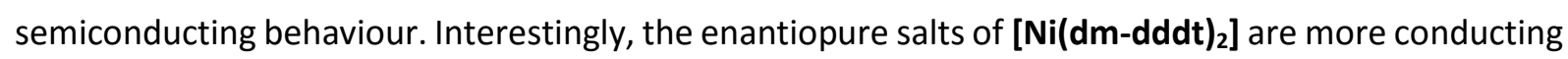
than the racemic form, very likely because of the structural disorder in the latter, while in [Ni(medddt $)_{2}$, not showing any disorder, the three compounds have comparable conductivity values. Highlighting the appropriateness of the chiral strategy in the metal dithiolene based conducting materials, this study represents a significant step forward towards the ultimate goal of reaching chiral single component metals and, possibly, superconductors. 


\section{Experimental}

\subsection{Materials and methods}

Reactions of complexation were carried out under Argon atmosphere using the Schlenk technique. All compounds were obtained as single crystals and characterized by X-ray diffraction measurement. Elemental analysis were recorded using Flash 2000 Fisher Scientific Thermo Electron analyzer.

\subsection{Synthesis}

Synthesis of (TBA)[Ni(S-me-dddt $)_{2}$ ]. Sodium methoxide $(0.50 \mathrm{mmol}, 27 \mathrm{mg})$ was added to a solution of (S)-5-methyl-5,6-dihydro-1,3-dithiolo-1,4-dithiin-2-one $(0.20 \mathrm{mmol}, 44 \mathrm{mg})$ in dry methanol $(15 \mathrm{~mL})$. After 2 hours, $\mathrm{NiCl}_{2} .6 \mathrm{H}_{2} \mathrm{O}(0.10 \mathrm{mmol}, 24 \mathrm{mg})$ was added and the reaction was left to stir for 1 hour. Tetrabutylammonium bromide $(0.10 \mathrm{mmol}, 32 \mathrm{mg})$ was finally added to the mixture. After 1 hour, the solvent was reduced under pressure. The crude product was washed with diethylether and dissolved in dichloromethane. Single crystals were obtained by slow evaporation of the solvent at room temperature.

Synthesis of (TBA)[Ni(R-me-dddt) ${ }_{2}$ ]. This compound was prepared in a similar manner to (TBA) [Ni(Sme-dddt $)_{2}$ ] starting from $(R)$-5-methyl-5,6-dihydro-1,3-dithiolo-1,4-dithiin-2-one (0.20 mmol, $\left.44 \mathrm{mg}\right)$.

Synthesis of (TBA)[Ni(S-me-dddt)( $R$-me-dddt)]. This compound was prepared in two distinct ways: 1) in a similar manner to (TBA)[Ni(S-me-dddt) ${ }_{2}$ ] starting from ( $\left.r a c\right)$-5-methyl-5,6-dihydro-1,3-dithiolo-1,4dithiin-2-one (0.20 mmol, $44 \mathrm{mg})$. 2) Crystalline powders of (TBA)[Ni(S-me-dddt) ${ }_{2}$ ] $(0.01 \mathrm{mmol}, 10 \mathrm{mg}$ ) and (TBA)[Ni(R-me-dddt $\left.)_{2}\right](0.01 \mathrm{mmol}, 10 \mathrm{mg})$ were dissolved in a dichloromethane solution $(5 \mathrm{~mL})$. Single crystals of the racemic compound were obtained by slow evaporation of the solvent at room temperature.

Synthesis of (TBA)[Ni(S,S-dm-dddt)(R,R-dm-dddt)]. This compound was prepared in two distinct ways: 1) in a similar manner to (TBA) [Ni(S-me-dddt) 2 ] starting from ( $\mathrm{rac}$-5,6-methyl-5,6-dihydro-1,3-dithiolo1,4-dithiin-2-one (0.20 mmol, $47 \mathrm{mg})$. 2) Crystalline powders of (TBA)[Ni(S,S-dm-dddt) $)_{2}$ (0.01 mmol, $10 \mathrm{mg})$ and $(\mathrm{TBA})\left[\mathrm{Ni}(R, R-\mathrm{dm} \text {-dddt })_{2}\right](0.01 \mathrm{mmol}, 10 \mathrm{mg})$ were dissolved in a dichloromethane solution $(5 \mathrm{~mL})$. Single crystals of the racemic compound were obtained by slow evaporation of the solvent at room temperature.

Synthesis of (TMA) [Ni(S,S-dm-dddt) $)_{2}$. This compound was prepared in a similar manner to (TBA) [Ni(Sme-dddt $)_{2}$ ] starting from $(S, S)$-5,6-dimethyl-5,6-dihydro-1,3-dithiolo-1,4-dithiin-2-one $(0.20 \mathrm{mmol}, 47$ $\mathrm{mg}$ ) and tetramethylammonium chloride $(0.10 \mathrm{mmol}, 11 \mathrm{mg})$.

Synthesis of $(\mathrm{TMA})\left[\mathbf{N i}(\boldsymbol{R}, \boldsymbol{R}-\mathbf{d m}-\mathbf{d d d t})_{2}\right]$. This compound was prepared in a similar manner to $(\mathrm{TMA})\left[\mathrm{Ni}(S, S-d m \text {-dddt })_{2}\right]$ starting from $(R, R)$-5,6-dimethyl-5,6-dihydro-1,3-dithiolo-1,4-dithiin-2-one (0.20 mmol, $47 \mathrm{mg}$ ).

Synthesis of (TMA)[Ni(S,S-dm-dddt) $(R, R-d m-d d d t)]$. This compound was prepared in a similar manner to (TMA)[Ni(S,S-dm-dddt) $)_{2}$ starting from ( $\mathrm{rac}$ )-5,6-dimethyl-5,6-dihydro-1,3-dithiolo-1,4-dithiin-2-one $(0.20 \mathrm{mmol}, 47 \mathrm{mg})$. 
Synthesis of [Ni(S-me-dddt) $)_{2}$. Tetrabutylammonium hexafluorophosphate $(0.13 \mathrm{mmol}, 50 \mathrm{mg})$ was dissolved in a mixture of dichloromethane/acetonitrile $(6 / 6 \mathrm{~mL})$. Half of the solution was used to dissolve $10 \mathrm{mg}$ of (TBA)[Ni(S-me-dddt $)_{2}$. The first solution was placed in the cathodic compartment of an electrocrystallization cell and the second one in the anodic compartment. Single crystals were obtained on the anode electrode by applying a current density of $0.5 \mu \mathrm{A}$ at room temperature after one week.

Synthesis of [ $\mathbf{N i}(\boldsymbol{R} \text {-me-dddt })_{2}$ ]. Single crystals of this compound were obtained in a similar manner to [Ni(S-me-dddt $\left.)_{2}\right]$ starting from (TBA)[Ni(R-me-dddt $\left.)_{2}\right](0.01 \mathrm{mmol}, 10 \mathrm{mg})$.

Synthesis of [Ni(S-me-dddt)(R-me-dddt)]. Single crystals of this compound were obtained in a similar manner to [Ni(S-me-dddt) ${ }_{2}$ ] starting from (TBA)[Ni(S-me-dddt)(R-me-dddt)] $(0.01 \mathrm{mmol}, 10 \mathrm{mg})$.

Synthesis of [Ni(S,S-dm-dddt) $)_{2}$. Single crystals of this compound were obtained in a similar manner to $\left[\mathrm{Ni}(S-\mathrm{me}-\mathrm{dddt})_{2}\right]$ starting from $(\mathrm{TBA})\left[\mathrm{Ni}(\mathrm{S}, \mathrm{S}-\mathrm{dm}-\mathrm{dddt})_{2}\right](0.01 \mathrm{mmol}, 10 \mathrm{mg})$.

Synthesis of [Ni(S,S-dm-dddt)(R,R-dm-dddt)]. Single crystals of this compound were obtained in a similar manner to [Ni(S-me-dddt) ${ }_{2}$ ] starting from (TBA)[Ni(S,S-dm-dddt)( $R, R$-dm-dddt)] $(0.01 \mathrm{mmol}, 10$ $\mathrm{mg})$.

\subsection{DFT and TD-DFT calculations}

All DFT and TD-DFT calculations have been performed with the Gaussian 09 program $^{54}$ using the DFT method with the hybrid PBEO functional (with 25\% of exact exchange) and the augmented and polarized Ahlrichs triple-zeta basis set (noted TZVP). ${ }^{55}$ Default parameters were used. First, the gasphase ground state geometries were optimized starting from the X-ray diffraction data. The 15 lowlying excited-states energies were then determined for the gas-phase by a linear response TD-DFT method with the same parameters. The calculated spectra have been enlarged by a Gaussian shape [full width at half-maximum (FWHM) $=3000 \mathrm{~cm}^{-1}$ ]. The full reports of the computational results included in the supplementary data were prepared with quchemreport, a homemade python program based on cclib. ${ }^{56,57}$

\subsection{X-Ray structure determinations}

Single crystals were mounted on glass fibre loops using a viscous hydrocarbon oil to coat the crystal and then transferred directly to the cold nitrogen stream. Data collection were performed at $150 \mathrm{~K}$ on an Agilent Supernova with Cu- $K_{\alpha}(\lambda=1.54184 \AA$ A). The structures were solved by direct methods with the SIR97 program and refined all $F^{2}$ values with the SHELXL-2016/4 program using the WinGX graphical user interface. All non-hydrogen atoms were refined anisotropically. All hydrogen atoms were placed in calculated positions and refined isotropically with a riding model. A summary of crystallographic data and structure refinements is given in Tables S1-S6. Crystallographic data for the seventeen structures have been deposited with the CCDC: $2048855((S, S)-\mathbf{2}), 2048856((R, R)-\mathbf{2})$, 2048857 ((rac)-2), 2048858 ((TBA)[Ni(S-me-dddt) $)_{2}$ ), 2048859 ((TBA)[Ni(R-me-dddt) $)_{2}$ ]), 2048860 ((TBA)[Ni(S-me-dddt)(R-me-dddt)]), $2048861 \quad((T B A)[N i(S$-me-dddt)(R-me-dddt)] scrambling), 2048862 ((TBA)[Ni(S,S-dm-dddt)(R,R-dm-dddt)]), 2048863 ((TBA)[Ni(S,S-dm-dddt)(R,R-dm-dddt)] scrambling), 2048864 ((TMA)[Ni(S,S-dm-dddt) $\left.\left.)_{2}\right]\right), 2048865$ ((TMA)[Ni(R,R-dm-dddt) 2$\left.]\right), 2048866$ ((TMA)[Ni(S,S-dm-dddt)(R,R-dm-dddt)]), 2048867 ([Ni(S-me-dddt) 2$]), 2048868$ ([Ni(R-me-dddt) $]$ ]), 2048869 ([Ni(S-me-dddt)(R-me-dddt)]), 2048870 ([Ni(S,S-dm-dddt) $]), 2048871$ ([Ni(S,S-dm- 
dddt)(R, $R$-dm-dddt)]). These data can be obtained free of charge from The Cambridge Crystallographic Data Centre.

\subsection{Conductivity measurements}

All single crystals were cut into an average size of $0.15 \times 0.02 \times 0.015 \mathrm{~mm}^{3}$ and used to measure highpressure resistivity. The sample was mounted in the DAC by using the same technique as that used for [Ni(ptdt $\left.)_{2}\right]{ }^{15}$ Electrical contacts were obtained by attaching four $\phi 10 \mu \mathrm{m}$ gold wires with gold paint. All samples were then encapsulated in a thin layer of epoxy and alumina. The diamond anvil with culet size of $0.7 \mathrm{~mm}$ and tension annealed stainless steel SUS301 were used for all samples, and the fourprobe DC method was used for all measurements. Daphne Oil 7373 was used as the pressure transmitting medium. The pressure was determined by the shift in the ruby fluorescence $R 1$ lines at room temperature. All samples were measured using the cryocooler helium compressor system (Sumitomo Heavy Industries, Ltd.) for cooling the DAC with a cooling rate of $1.5 \mathrm{~K} / \mathrm{min}$.

\subsection{Band structure calculations}

The first-principles calculations were carried out using a numeric atomic orbitals density functional theory (DFT) approach ${ }^{58,59}$ developed for efficient calculations in large systems and implemented in the SIESTA code. ${ }^{60,61,62,63}$ We used the generalized gradient approximation (GGA) to DFT and, in particular, the functional of Perdew, Burke, and Ernzerhof. ${ }^{64}$ To study the relative energies of states with localized electrons, band calculations for appropriate supercells have been undertaken. All calculations included a Hubbard correction term $U_{\text {eff }}=\mathrm{U}-\mathrm{J}=6.0 \mathrm{eV}$ for the sulfur $3 p$ states..$^{65}$ In previous work ${ }^{66}$ we have found that this $U$ term on sulfur atoms is needed for appropriately describing the electronic structure of TTF-TCNQ and related molecular solids where accurate experimental information on the bandwidth and charge transfer is available. Only the valence electrons are considered in the calculation, with the core being replaced by norm-conserving scalar relativistic pseudopotentials ${ }^{67}$ factorized in the Kleinman-Bylander form. ${ }^{68}$ We have used a split-valence double- $\zeta$ basis set including polarization orbitals with an energy shift of $10 \mathrm{meV} .{ }^{69}$ The energy cutoff of the real space integration mesh was 300 Ry. The Brillouin zone was sampled using grids ${ }^{70}$ of $(9 \times 9 \times 9) k$-points for the neutral systems, sets of $(9 \times 9 \times 9)$ and $(5 \times 9 \times 9) k$-points for the single-cell and double-cell calculations for (TBA)[Ni(dm-dddt) ${ }_{2}$ ] and sets of $(5 \times 5 \times 5)$ and $(5 \times 3 \times 5) k$-points for the single-cell and double-cell calculations for $(\mathrm{TMA})\left[\mathrm{Ni}(\mathbf{d m}-\mathbf{d d d t})_{2}\right]$. The crystal structures at $150 \mathrm{~K}$ (see ESI) were used for the computations.

Tight-binding band structure calculations of the extended Hückel type ${ }^{71}$ were also carried out for the

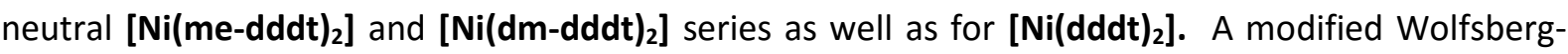
Helmholtz formula was used to calculate the non-diagonal $H_{\mu v}$ values. ${ }^{72}$ All valence electrons were taken into account in the calculations and the basis set consisted of Slater-type orbitals of double- $\zeta$ quality for $\mathrm{C} 2 \mathrm{~s}$ and $2 \mathrm{p}, \mathrm{S} 3 \mathrm{~s}$ and $3 p$ and of single- $\zeta$ quality for $\mathrm{H} 1 \mathrm{~s}$. The ionization potentials, contraction coefficients and exponents were taken from previous work. ${ }^{73}$

\section{Conflicts of interest}

There are no conflicts to declare. 


\section{Acknowledgements}

This work was supported in France by the National Agency for Research (ANR, Project 15-CE29-000601 ChiraMoICo), the CNRS and the University of Angers. Work in Spain was supported by MICIU (Spain) through Grants PGC2018-096955-B-C44 and PGC2018-093863-B-C22, and by Generalitat de Catalunya (2017SGR1506 and 2017SGR1289). E. C. acknowledges support of the Spanish MICIU through the Severo Ochoa FUNFUTURE (CEX2019-000917-S) Excellence Centre distinction and P. A. from the Maria de Maeztu Units of Excellence Program (MDM-2017-0767). The work in Japan was supported by JSPS KAKENHI (Grants No. 16H06346).

\section{Notes and references}

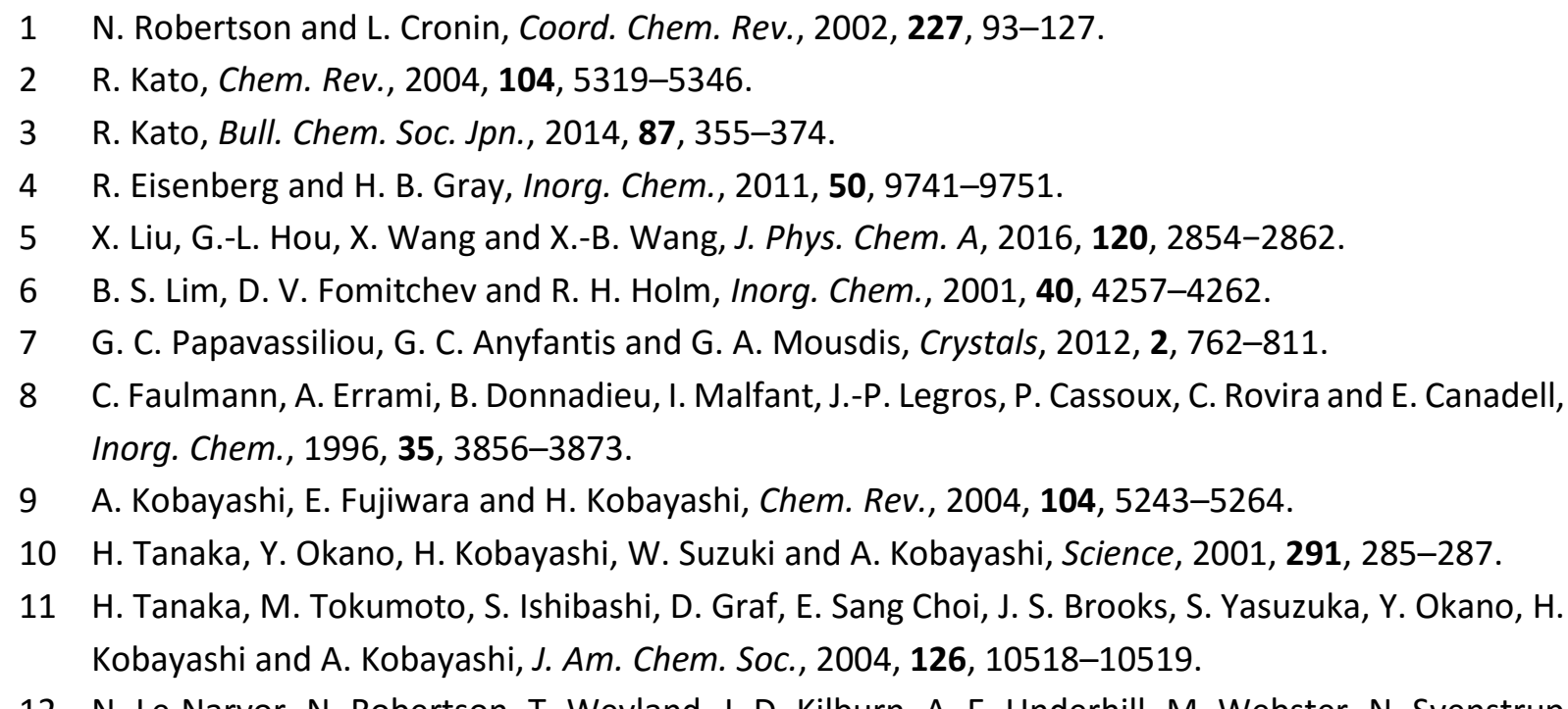

12 N. Le Narvor, N. Robertson, T. Weyland, J. D. Kilburn, A. E. Underhill, M. Webster, N. Svenstrup and J. Becher, Chem. Commun., 1996, 1363-1364.

13 A. Kobayashi, H. Tanaka, M. Kumasaki, H. Torii, B. Narymbetov and T. Adachi, J. Am. Chem. Soc., 1999, 121, 10763-10771.

14 M. Sasa, E. Fujiwara, A. Kobayashi, S. Ishibashi, K. Terakura, Y. Okano, H. Fujiwara and H. Kobayashi, J. Mater. Chem., 2005, 15, 155-163.

15 H. Cui, J. S. Brooks, A. Kobayashi and H. Kobayashi, J. Am. Chem. Soc., 2009, 131, 6358-6359.

16 H. Cui, H. Kobayashi, S. Ishibashi, M. Sasa, F. Iwase, R. Kato and A. Kobayashi, J. Am. Chem. Soc., 2014, 136, 7619-7622.

17 H. Cui, T. Tsumuraya, T. Miyazaki, Y. Okano and R. Kato, Eur. J. Inorg. Chem., 2014, 3837-3840.

18 H. Hachem, H. Cui, T. Tsumuraya, R. Kato, O. Jeannin, M. Fourmigué and D. Lorcy, J. Mater. Chem. C, 2020, 8, 11581-11592.

19 H. Cui, T. Tsumuraya, H. H.-M. Yeung, C. S. Coates, M. R. Warren and R. Kato, Molecules, 2019, 24, 1843.

20 A. Filatre-Furcate, N. Bellec, O. Jeannin, P. Auban-Senzier, M. Fourmigué, A. Vacher and D. Lorcy, Inorg. Chem., 2014, 53, 8681-8690. 
21 R. Kato, H. Cui, T. Tsumuraya, T. Miyazaki and Y. Suzumura, J. Am. Chem. Soc., 2017, 139, 1770-1773.

22 T. Tsumuraya, R. Kato and Y. Suzumura, J. Phys. Soc. Jpn., 2018, 87, 113701.

23 R. Kato, H. Cui, T. Minamidate, H. H.-M. Yeung and Y. Suzumura, J. Phys. Soc. Jpn., 2020, 89, 124706.

24 F. Pop, N. Zigon and N. Avarvari, Chem. Rev., 2019, 119, 8435-8478.

25 N. Avarvari and J. D. Wallis, J. Mater. Chem., 2009, 19, 4061-4076.

26 V. Krstic, S. Roth, M. Burghard, K. Kern and G. L. J. A. Rikken, J. Chem. Phys., 2002, 117, 1131511319.

27 F. Pop, P. Auban-Senzier, E. Canadell, G. L. J. A. Rikken and N. Avarvari, Nat. Commun., 2014, 5, 3757.

28 G. L. J. A. Rikken and N. Avarvari, Phys. Rev. B, 2019, 99, 245153.

29 C. Réthoré, N. Avarvari, E. Canadell, P. Auban-Senzier and M. Fourmigué, J. Am. Chem. Soc., 2005, 127, 5748-5749.

30 A. M. Madalan, C. Réthoré, M. Fourmigué, E. Canadell, E. B. Lopes, M. Almeida, P. Auban-Senzier and N. Avarvari, Chem. Eur. J., 2010, 16, 528-537.

31 F. Pop, S. Laroussi, T. Cauchy, C. J. Gómez-García, J. D. Wallis and N. Avarvari, Chirality, 2013, 25, 466-474.

32 F. Pop, P. Auban-Senzier, A. Frąckowiak, K. Ptaszyński, I. Olejniczak, J. D. Wallis, E. Canadell and N. Avarvari, J. Am. Chem. Soc., 2013, 135, 17176-17186.

33 F. Pop, P. Auban-Senzier, E. Canadell and N. Avarvari, Chem. Commun., 2016, 52, 12438-12441.

34 H. Kisch, B. Eisen, R. Dinnebier, K. Shankland, W. I. F. David and F. Knoch, Chem. Eur. J., 2001, 7, 738-748.

35 J. Lieffrig, O. Jeannin, P. Auban-Senzier and M. Fourmigué, Inorg. Chem., 2012, 51, 7144-7152.

36 F. Pop and N. Avarvari, Coord. Chem. Rev. 2017, 346, 20-31.

37 N. Mroweh, C. Mézière, M. Allain, P. Auban-Senzier, E. Canadell and N. Avarvari, Chem. Sci., 2020, 11, 10078-10091.

38 N. Mroweh, C. Mézière, F. Pop, P. Auban-Senzier, P. Alemany, E. Canadell and N. Avarvari, Adv. Mater., 2020, 32, 2002811.

39 N. Mroweh, P. Auban-Senzier, N. Vanthuyne, E. Canadell and N. Avarvari, J. Mater. Chem. C, 2019, 7, 12664-12673.

40 D. G. Branzea, F. Pop, P. Auban-Senzier, R. Clérac, P. Alemany, E. Canadell and N. Avarvari, J. Am. Chem. Soc., 2016, 138, 6838-6851.

41 G. C. Papavassiliou, G. A. Mousdis and A. Papadima, Z. Naturforsch. B, 2000, 55, 231-232.

42 J. D. Dunitz, A. Karrer and J. D. Wallis, Helv. Chim. Acta, 1986, 69, 69-70.

43 S. Matsumiya, A. Izuoka, T. Sugawara, T. Taruishi and Y. Kawada, Bull. Chem. Soc. Jpn, 1993, 66, 513-522.

44 T. Cauchy, F. Pop, J. Cuny and N. Avarvari, Chimia, 2018, 72, 389-393.

45 S. Yang, F. Pop, C. Melan, A. C. Brooks, L. Martin, P. Horton, P. Auban-Senzier, G. L. J. A. Rikken, N. Avarvari and J. D. Wallis, CrystEngComm, 2014, 16, 3906-3916.

46 J. D. Wallis and J. D. Dunitz, Acta Cryst., 1988, C44, 1037-1039.

47 G. Soras, N. Psaroudakis, M. J. Manos, A. J. Tasiopoulos, D. G. Liakos and G. A. Mousdis, Polyhedron, 2013, 62, 208-217.

48 J. H. Welch, R. D. Bereman, P. Singh, D. Haase, W. E. Hatfield and M. L. Kirk, Inorg. Chim. Acta, 1989, 162, 89-96. 
49 F. Pop, P. Batail and N. Avarvari, Crystals, 2016, 6, 8.

50 M. Atzori, F. Pop, P. Auban-Senzier, R. Clérac, E. Canadell, M. L. Mercuri and N. Avarvari, Inorg. Chem., 2015, 54, 3643-3653.

51 M.-H. Whangbo, J. M. Williams, P. C. W. Leung, M. A. Beno, T. J. Emge and H.-H. Wang, Inorg. Chem., 1985, 24, 3500-3502.

52 For two-orbital systems like the metal bis(dithiolene) solids, the crossed HOMO-LUMO terms can sometimes be also important but except when otherwise stated, it is not essential for the present qualitative considerations.

53 N. W. Ashcroft and N. D. Mermin, Solid State Physics, Holt, Rinehart and Winston, Philadelphia, 1976.

54 M. J. Frisch et al., Gaussian $\sim 09$ Revision D.01.

55 A. Schäfer, C. Huber and R. Ahlrichs, J. Chem. Phys., 1994, 100, 5829-5835.

56 T. Cauchy and B. Da Mota, quchemreport. A python program for control quality and automatic generation of quantum chemistry results, University of Angers, 2020.

57 N. M. O'boyle, A. L. Tenderholt and K. M. Langner, J. Comput. Chem., 2008, 29, 839-845.

58 P. Hohenberg and W. Kohn, Phys. Rev., 1965, 136, B864-B871.

59 W. Kohn and L. J. Sham, Phys. Rev., 1965, 140, A1133-A1138.

60 J. M. Soler, E. Artacho, J. D. Gale, A. García, J. Junquera, P. Ordejón and D. Sánchez-Portal, J. Phys.: Condens. Matter., 2002, 14, 2745-2779.

61 E. Artacho, E. Anglada, O. Diéguez, J. D. Gale, A. García, J. Junquera, R. D. Martin, P. Ordejón, J. M. Pruneda, D. Sánchez-Portal and J. M. Soler, J. Phys.: Condens. Matter., 2008, 20, 064208.

62 A. García, N. Papior, A. Akhtar, E. Artacho, V. Blum, E. Bosoni, P. Brandimarte, M. Brandbyge, J. I. Cerdá, F. Corsetti, R. Cuadrado, V. Dikan, J. Ferrer, J. Gale, P. García-Fernández, V. M. GarcíaSuárez, S. García, G. Huhs, S. Illera, R. Korytar, P. Koval, I. Lebedeva, L. Lin, P. López-Tarifa, S. G. Mayo, S. Mohr, P. Ordejón, A. Postnikov, Y. Pouillon, M. Pruneda, R. Robles, D. Sánchez-Portal, J. M. Soler, R. Ullah, V. Wen-zhe Yu and J. Junquera, J. Chem. Phys., 2020, 152, 204108.

63 For more information on the SIESTA code visit: http://departments.icmab.es/leem/siesta/

64 J. P. Perdew, K. Burke and M. Ernzerhof, Phys. Rev. Lett., 1966, 77, 3865-3868.

65 S. L. Dudarev, G. A. Botton, S. Y. Savrasov, C. J. Humphreys and A. P. Sutton, Phys. Rev. B: Condens. Matter Mater. Phys., 1998, 57, 1505-1509.

66 Y. Kiyoka, I.-R. Jeon, O. Jeannin, M. Beau, T. Kawamoto, P. Alemany, E. Canadell, T. Mori and M. Fourmigué, Phys. Chem. Chem. Phys., 2019, 21, 22639-22646.

67 N. Troullier and J. L. Martins, Phys. Rev. B, 1991, 43, 1993-2006.

68 L. Kleinman and D. M. Bylander, Phys. Rev. Lett., 1982, 48, 1425-1428.

69 E. Artacho, D. Sánchez-Portal, P. Ordejón, A. García and J. M. Soler, Phys. Stat. Sol. (b), 1999, 215, 809-817.

70 H. J. Monkhorst and J. D. Pack, Phys. Rev. B, 1976, 13, 5188-5192.

71 M.-H. Whangbo and R. Hoffmann, J. Am. Chem. Soc., 1978, 100, 6093-6098.

72 J. H. Ammeter, H.-B. Bürgi, J. Thibeault and R. Hoffmann, J. Am. Chem. Soc., 1978, 100, 36863692.

73 A. Pénicaud, K. Boubekeur, P. Batail, E. Canadell, P. Auban-Senzier and D. Jérome, J. Am. Chem. Soc., 1993, 115, 4101-4112. 